\title{
Formation damage induced by wax deposition: laboratory investigations and modeling
}

\author{
M. S. Sandyga ${ }^{1} \cdot$ I. A. Struchkov ${ }^{2}$ (D) M. K. Rogachev ${ }^{1}$
}

Received: 12 January 2020 / Accepted: 3 June 2020 / Published online: 12 June 2020

(c) The Author(s) 2020

\begin{abstract}
There are oil fields, wherein favorable conditions for the formation damage induced by wax deposition are created during production. The damage can be expressed by a decrease in porosity and permeability and a reduction in the drainage area. There are only a few unconventional fields, and this makes them unique. To prevent this complication, it is necessary to control the field production. Assuming the presence of such problem, the conventional reserves may turn into difficult oil reserves whose production is problematic, which will compromise the project profitability. The key to the problem is associated with the experimental procedure and research conditions for investigation wax crystallization in oil, being the subject of this paper. The authors showed that the use of WAT measurement technique in an open measuring system is not enough to control wax deposition in the reservoir pore volume. Based on the results of the flooding technique and micro-computed tomography, a digital core, that allows to simulate fluid flow in the porous medium of the core before and after formation damage, has been created. The calculation of the change in the thermal field around the injection well over time, according to the extended Lauwerier's concept, has been carried out. WAT of a wax-bearing solution was measured by the rheology method using an open measuring system (plate-to-plate measuring system under atmospheric pressure), and the dependence of viscosity versus temperature was obtained during experimental studies. The temperature was decreased from 60 to $10{ }^{\circ} \mathrm{C}$ at a cooling rate of $1{ }^{\circ} \mathrm{C} / \mathrm{min}$. The experiment was carried out at atmospheric pressure and a shear rate of $5 \mathrm{~s}^{-1}$. Also, filtration technique and micro-computed tomography were used. The dependence of the pressure gradient versus temperature and the pore throat diameter distribution functions for the initial core and core with organic scales were obtained. The flooding experiment was carried out at a constant flow rate of $0.5 \mathrm{~cm}^{3} / \mathrm{min}$ and confining pressure of $4.1 \mathrm{MPa}$. The temperature was decreased from 40 to $33^{\circ} \mathrm{C}$ at a cooling rate of $1{ }^{\circ} \mathrm{C} / \mathrm{h}$. The inflection points on the curves viscosity versus temperature and pressure gradient versus temperature confirm the WAT. The results of the laboratory experiments showed that WAT, measured by the rheology method is $3-4{ }^{\circ} \mathrm{C}$ lower than WAT, measured by the flooding technique. The results of the micro-computed tomography showed that initial porosity decreased from 9.0 to $2.1 \%$ as a result of wax deposition. The pore throats with diameters from 20 to $70 \mu \mathrm{m}$ are involved in the clogging with wax. The calculation results confirmed the possibility of cooling the near-wellbore area of injector to a temperature equal to WAT and the cold front movement to the producing wells. The production profiles calculated based on the models of porosity and permeability reduction, showed that wax deposition in the near-wellbore area can cause a significant decrease in the productivity index. An effective remediation technology for injection wells was proposed.
\end{abstract}

Keywords Rheology $\cdot$ Micro-computed tomography $\cdot$ Wax appearance temperature $\cdot$ Organic scales $\cdot$ Formation damage

\section{Introduction}

The production of waxy oil is usually associated with the formation of organic scales in downhole equipment and pro-

I. A. Struchkov

StruchkovIA@gmail.com

1 Saint-Petersburg Mining University, Saint Petersburg, Russia

2 LLC Tyumen Petroleum Research Center, Tyumen, Russia duction facilities. Rheology, filtration, and light scattering methods, as well as nuclear magnetic resonance, are widely used to study this issue (Elsharkawy et al. 2000; Karan et al. 2000; Jiang et al. 2001; Paso et al. 2009; Jiang et al. 2014;

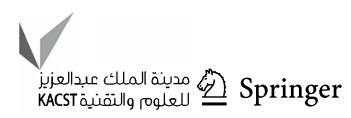


Chen et al. 2014; Huang et al. 2016). However, processes that occur in the reservoir and the least understood are the matters of outstanding interest. Pressure and temperature are the key parameters, affecting the formation of organic scales in the pore volume of the reservoir. The use of the reservoir pressure maintenance system during the field production makes it possible to maintain the reservoir energy at a target level. However, in most cases, the temperature of the injected water is not controlled. Therefore, resulting from the injection of large volume of cold water, a decrease in reservoir temperature is observed (Muslimov et al. 1968; Mingareev et al. 1968; Mezzomo and Rabinovitz 2001; Rocha et al. 2003). The problem of clogging the pore volume of the near-wellbore area with wax resulting from a decrease in temperature is described by Borisov et al. (2018), Yusupova et al. (2016). The flooding experiments are carried out by the authors, proved that a decrease in temperature leads to core permeability decrease due to the formation of wax crystals. Moreover, according to some scientists, the formation of organic scales in the porous medium can already begin at the well completion, resulting from lost circulation of cold process fluids (Newberry and Barker 1985; Orodu and Tang 2014).

One of the main properties of reservoir oil, taken into account when selecting techniques for preventing the wax formation in the near-wellbore area (Khalil et al. 1997), is the wax appearance temperature (WAT). Oil is the complex multicomponent dispersed system, and the change in its composition during field production significantly affects the WAT and other properties of oil. Even using all the existing research techniques and laboratory equipment, the researcher is unable to fully describe all possible combinations of compositions, as well as to evaluate the contribution of each component, taken separately, to the change in its properties. Due to the complexity of a comprehensive analysis of the impact of various oil components on the wax formation, this paper presents research techniques and the results of modeling the simplified systems-wax-bearing solutions.

\section{Scope of WAT investigation techniques}

\section{American society for testing and materials (ASTM) visual methods}

The ASTM visual methods are based on the visual observation of the first wax crystals in the test fluid. The fluid is stirred and cooled under prescribed conditions. The temperature at which the first wax crystals are visible is taken as WAT. The main disadvantages of this method include the low resolution, as well as the impossibility of visual observation of the wax crystals formation in dark solutions (Ijeomah et al. 2008; Uba et al. 2004). Therefore, this method can only be used for model solutions and transparent petroleum products.

\section{Cold finger}

Cold finger is a method for studying the freezing point of organic scales. The organic scales deposition is simulated on the metal walls of downhole and surface equipment by this method. The cooled metal rod (finger) imitates the inner wall of the pipe. A heated and stirred oil sample flushes it. When the rod temperature drops below WAT, wax deposition begins on its surface. The wax formation depends on many factors, such as the temperature of oil and the finger, the ratio of the finger surface to the volume of oil sample, temperature gradients and stirring rate (Dos Santos et al. 2004). This method is often used to estimate the efficiency of wax inhibitors and solvents.

\section{Viscometry methods}

Viscometry method is one of the most available, easy-to-use and cheap measuring techniques. In the oil industry, widely used are rotational viscometers, the measurement principle of which is based on the law of fluid flow in the system of concentric-cylinder (between coaxial cylinders), plate-toplate, and cone-plate (in the gap of the measuring system) type. One of the bodies remains stationary (stator, plate, etc.), the other, called the rotor of the rotational viscometer, rotates at a constant rotary speed. The rotational motion of the rotor is transmitted to the stator through the motion of a viscous medium. During the experiment, the measuring system measures the rotating torque of the rotor, which is a measure for the fluid viscosity. Using this research method, it is possible to measure with high accuracy the fluid viscosity fluctuation on change in temperature (Kok et al. 1996). WAT is determined by the inflection point of curve (the dependence of fluid viscosity on temperature). This is an indirect method of determining WAT, but it allows measuring WAT at high pressure (using closed measuring system of concentric-cylinder type).

\section{Differential-scanning calorimetry}

Differential-scanning calorimetry is a group of methods of physicochemical analysis that makes it possible to estimate WAT by measuring the heat of phase transition. The principle of experimental procedure for differential-scanning colorimetry is based on measuring temperature difference (heat flux) depending on the time between the studied sample and the calibration one. The studied sample is placed at the bottom of the measurement cell, and good thermal contact between the sample and the sensor must be provided. Then, the cell is tightly closed and measurement begins. 
Wax crystallization is accompanied by heat release, which is detected by the measuring system. Using this method, it is possible to measure with high accuracy the temperature of the phase transition in the studied fluid (Sherman et al. 2019; Vicky Kett et al. 2016). The method makes it possible to directly measure the enthalpy of wax crystallization. However, the drawback of this method is the impossibility of measuring WAT at high pressure and in the pore volume of rock (Drzeżdżon et al. 2018).

\section{Cross-polarized microscopy}

Cross-polarized microscopy is used to study fluids in polarized light (transmitted and reflected). In the oil industry, this kind of research is used to check for the presence of high molecular weight components (wax, asphaltenes) in oil and to measure the size of particles, formed by these components. In addition, this method is widely used to select the composition of organic scale inhibitors. The advantage of this method resides in the fact that it is a direct (optical) research method with a high resolution (capable of detecting particles up to $0.5 \mu \mathrm{m}$ in size) (Bacon et al. 2009). The drawbacks include the small volume of sample used for the study and inability to carry out the study at high pressure.

\section{Light transmittance}

The Light transmittance method makes it possible to determine the amount of precipitated wax in solution by measuring the light transmittance. Moreover, the accuracy of this method is higher for solutions with dark precipitates. In the oil industry, this method is used to determine the amount of wax crystals or asphaltenes and resins, precipitated in oil or model solutions (Uba et al. 2004). This method allows detecting wax crystals at the nucleation stage $(1 \mu \mathrm{m}$ in size $)$. This method also allows measuring WAT at high pressure.

\section{Ultrasonics}

Ultrasonic method is widely used for high wax oils with a high freezing point. The method is based on measuring the speed of ultrasonic waves passing through a studied fluid, which depends on its density. Wax crystals in oil dissipate acoustic energy, which allows detecting WAT. This method is sensitive to the size and amount of solids in oil (Jiang et al. 2014).

\section{A near-infrared (NIR) scattering}

A near-infrared (NIR) scattering method is widely used to measure WAT in oil and model solutions in dynamic conditions. The method is based on measuring the coefficient of infrared radiation absorption by the wax crystals, formed with a decrease in temperature. This method has a higher resolution than cross-polarized microscopy. NIR at a wavelength of $1100 \mathrm{~nm}$ can detect wax crystals smaller than $55 \mathrm{~nm}$ in size (Paso et al. 2009).

None of the above-mentioned methods allows measuring WAT in the pore volume of rock. The methods differ from each other in the sensitivity of measuring systems and the principle of operation.

Different methods for measuring WAT have different sensitivity. Each method should be used for specific conditions (stages of the organic scales deposition in the field). For example, if reservoir cooling is not observed or this process takes too long (meanwhile, the initial reservoir temperature is much higher than WAT), then only the rheology method can be used to control the wax formation, since wax crystallization in the pore volume of the reservoir should not occur. In this case, organic scales can form in tubing and flow lines. However, if the reservoir temperature drops below WAT, then both measuring systems should be used. When measuring WAT, an error of more than $3-4{ }^{\circ} \mathrm{C}$ can lead to losses in well productivity. Methods for well productivity remediation (bottom hole treatment) are more expensive and timeconsuming than methods for cleaning tubing and flow lines.

The objective of this paper is to show that the use of WAT measurement technique in an open measuring system is not enough to predict and control the formation of organic scales in the pore volume of rock. The data obtained will facilitate the efficiency of well operation in the fields with high wax content due to WAT control in the reservoir. The novelty of the paper is that the authors showed a significant difference between WAT in an open measuring system and WAT in the pore volume of rock. This fact is confirmed by the results of rheology, filtration, as well as tomography studies. The authors substantiated the necessity of applying a complex of laboratory methods, field survey and modeling to predict and control the formation damage induced by wax deposition. In addition, an uncertainty analysis of the simulation results on the input parameters obtained during laboratory experiments was carried out.

\section{The history and scope of the field production}

One field on which the wax formation in the near-wellbore area was noted is the Romashkinskoye field, a giant Russian oil field, located in the Republic of Tatarstan. The field, discovered in 1948, is the largest field in the Volga-Ural oil province. The average producing depth is 1750 meters, wherein the initial reservoir temperature fluctuates around $37^{\circ} \mathrm{C}$. The oil of the Romashkinskoye field is waxy (about $5 \%$ by weight), resin ( $16.5 \%$ by weight) with a density of $820 \mathrm{~kg} / \mathrm{m}^{3}$. The field was brought into production in 1952 . Due to the large size of the field, peripheral and marginal water flooding was ineffective and it was impossible to

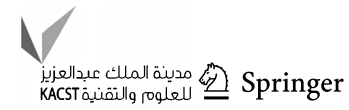


maintain the reservoir pressure at a target level. In the field for the first time in Russia, it was proposed a contour water flooding system. So, the field was cut into more than 20 blocks by rows of injection wells. However, the water intake capacity of some formations decreased over time. The organic scales formation is deemed to be one of the most challenging issues during oil production in the field. In the 50-60 s of the last century, during the period of maximum oil production, the workover period of some wells was equal about 3-4 h (Tronov and Guskova 1999). This field is one of the few in Russia, where the issue of wax deposition in the pore volume was subjected to consideration, and a long-term survey of changes in the reservoir temperature associated with the injection of large volume of cold water was carried out. The use of observation wells made it possible to study the impact of cold water injection on the change in the temperature field in the reservoir during 6-7 years of production (Muslimov et al. 1968; Mingareev et al. 1968).

The first significant decrease in reservoir temperature to $23{ }^{\circ} \mathrm{C}$ was recorded in 1966 in a well, located at a distance of $250 \mathrm{~m}$ from the injection well. The well was operated with a production rate of 70-110 t/day. Water injection was begun in 1960. The water intake capacity of the injector varied in the range of $700-1800 \mathrm{~m}^{3} /$ day at a formation thickness of about $10 \mathrm{~m}$. The temperature of water opposite the formation varied in winter from 7 to $12{ }^{\circ} \mathrm{C}$, and in summer from 18 to $25^{\circ} \mathrm{C}$. In 5 months after the start of water injection, a four-time flushing of the pore volume with water was implemented, and a producing well with water cut of $50 \%$ was converted into the observation category. As an example, two more producing wells, drilled at a distance of 390 and $600 \mathrm{~m}$ from the injection row of wells can be specified. During 6 years, the temperature in the first well decreased from 37 to $26-27^{\circ} \mathrm{C}$. In the area of the second well, the reservoir was flushed with 1-2 pore volumes of water, wherein the downhole temperature decreased by only $1-2{ }^{\circ} \mathrm{C}$. The first well production rate was 3 times lower than the second well production rate ( $23 \mathrm{t} /$ day vs. $60-80 \mathrm{t} /$ day). According to the authors, a decrease in the productivity of wells in cooled formation could be caused both by a decrease in oil mobility and by wax deposition. Moreover, undeveloped formations, located above and below the formations being in production, can be cooled, which can also cause difficulties during their development (Muslimov et al. 1968; Mingareev et al. 1968).

\section{Materials and methods}

\section{Measurement of the wax appearance temperature in wax-in-kerosene solution by rheology method}

\section{Test samples}

In all laboratory investigations a wax-in-kerosene solution was examined. Materials, wax concentration as well as a method for preparing solutions are described below.

To measure WAT, a wax-bearing solution was prepared with wax concentration of $20 \%$ wt. The estimated volume of kerosene was poured into a quartz glass cuvette. Then, the desired amount of solid wax was added. After that, the cuvette was covered with a sealed cap and heated in a water bath to a temperature of $52-58{ }^{\circ} \mathrm{C}$ (until the wax was completely dissolved). In order to accelerate wax dissolution, the sealed cap was periodically removed and the solution was stirred with a glass rod, then the cap was returned in place. This solution was prepared immediately before the performance of the experiment.

There was used kerosene, which is recognized by the ASTM International standard specification D-3699-78 as grade $1-\mathrm{K}$ (less than $0.04 \%$ sulfur by weight) in this study. Kerosene is produced from the fractional distillation of petroleum between 150 and $290{ }^{\circ} \mathrm{C}$ at atmospheric pressure, resulting in a mixture of hydrocarbons with carbon number from C9 to C16. Density of kerosene is $0.78-0.81 \mathrm{~g} / \mathrm{cm}^{3}$, the pour point standardized at $-47^{\circ} \mathrm{C}$ and gel point is around $-40{ }^{\circ} \mathrm{C}$ (Shepherd et al. 2000). Wax is derived from petroleum and presented by the mixture of hydrocarbons with carbon number from 20 to 40 . The melting point of wax is approximately about $52^{\circ} \mathrm{C}$, so it is solid at ambient temperature. Density of wax is around $900 \mathrm{~kg} / \mathrm{m}^{3}$ (Ferris et al. 1931).

\section{The laboratory apparatus}

WAT in a wax-bearing solution was measured by rheology method using an automated viscosity analyzer-a rheometer at atmospheric pressure in a plate-to-plate measuring system (open measuring system). The resolution of this system is up to $0.1 \mu \mathrm{Pa}^{*} \mathrm{~s}$. Tests with solution were carried out under the following conditions: no slippage, steadystate laminar flow, no chemical reactions in the sample during tests. During the experiment cooling and heating of the solution were carried out using the Peltier element. 


\section{The experimental procedure}

After preparing, the solution was placed in a measuring system and kept at a temperature of $60{ }^{\circ} \mathrm{C}$ for a few minutes. Then an experiment was carried out at a shear rate of $5 \mathrm{~s}^{-1}$ and a cooling rate of $1{ }^{\circ} \mathrm{C} / \mathrm{min}$. The solution was cooled from 60 to $10{ }^{\circ} \mathrm{C}$, while the measuring system recorded the solution temperature and viscosity. WAT in the solution was defined by shape of the viscosity curve (dependence of the solution viscosity on temperature).

\section{Measurement of the wax appearance temperature in porous medium}

The laboratory apparatus To measure WAT in the solution in the pore volume of rock, a flooding experiment was carried out. This method allows obtaining the dependence of the pressure gradient on temperature, wherein WAT in the solution was defined by shape of the curve.

The unit consists of two two-cylinder pumps, two piston-type accumulators (for the studied fluids) and a core holder. Fluid pressure is measured and controlled via pressure gauges, located inlet and outlet of the core holder. All equipment of the unit is located in an air oven. The filtration unit allows to create confining pressure and pore pressure up to $70 \mathrm{MPa}(10,000 \mathrm{psi})$ with an accuracy of $0.01 \mathrm{psi}$, as well as a temperature up to $150{ }^{\circ} \mathrm{C}$ with an accuracy of $\pm 0.5^{\circ} \mathrm{C}$.

The experimental procedure Firstly, a sand core sample with a diameter of $3 \mathrm{~cm}$ and a length of $5 \mathrm{~cm}$ was extracted with an alcohol-benzene mixture at the ratio of 1:2. Then the core was placed in an air oven and dried until reaching a constant weight at a temperature of $105^{\circ} \mathrm{C}$.

To carried out the flooding experiment, two fluids were prepared: a wax-bearing solution with wax concentration of $20 \% \mathrm{wt}$ and model of the reservoir water.

The experimental technique consists of 4 stages:

1. the core was saturated with model of the reservoir water under vacuum;

2. residual water saturation in the core was created by the centrifugation technique;

3. a core sample was placed in a filtration unit. The initial temperature in the air oven was set equal to $40{ }^{\circ} \mathrm{C}$ (this is $10^{\circ} \mathrm{C}$ higher than WAT in the solution), the confining pressure was set equal to 4.1 MPa. Filtration of a wax-bearing solution was performed at a constant flow rate $\left(0.5 \mathrm{~cm}^{3} / \mathrm{min}\right)$, with a cooling rate of $1{ }^{\circ} \mathrm{C} / \mathrm{h}$. The temperature was decreased from 40 to $33{ }^{\circ} \mathrm{C}$. The pressure gradient, temperature and volume of the injected solution were measured;

4. filtration of model of reservoir water was performed at a constant flow rate $\left(0.5 \mathrm{~cm}^{3} / \mathrm{min}\right)$, at a temperature of
$33{ }^{\circ} \mathrm{C}$, until the kerosene recovery terminated, to remove kerosene with wax that was not involved in the deposition in the porous medium.

\section{Micro-computed tomography}

The laboratory apparatus Micro-computed tomography is a nondestructive method for studying the absorbing capacity of rocks. The method is based on different absorbing capacities of $\mathrm{X}$-radiation by various minerals that compose the rock. The sample was fixed in a holder inside the tomograph cabin. After the start of scanning, the holder with the sample was rotated around the vertical axis by $360^{\circ}$ at a specified rotary speed, ensuring a series of X-ray images to be recorded. X-radiation passes through the sample, with loss in power proportionally to the rock density, and is recorded by the receiver. From the data obtained, twodimensional images of the sample in gray scale are created. In such images, brightness characterizes the attenuation of $\mathrm{X}$-radiation (Hounsfield scale) by the rock due to the photoelectric effect and Compton scattering. Then, the images are reconstructed into a three-dimensional sample.

To perform the studies, an X-ray computer micro-tomograph was used. This device has a radiation source with a power of $40-130 \mathrm{kV}$, with a resolution of up to $7-8 \mu \mathrm{m}$.

The experimental procedure The micro-computed tomography technique consists of three stages:

At the first stage, the tomograph was calibrated to wax. A piece of solid wax was scanned and the Hounsfield unit (HU) was measured to be -600 . The possibility of detecting wax in the pore volume of the rock was then evaluated. Toward this core sample was prepared and hole with a diameter of $3.5 \mathrm{~mm}$ was drilled throughout the length of the core. The core was scanned and the volume of the hole was measured, after that the hole was filled with melted wax, allowed time to solidify, and scanned again. The discrepancy between the drilled hole volume obtained by the primary scanning and the wax volume obtained by the second scanning was 3-4\%. The results are shown in Fig. 1 ( $a-$ core with hole, $b-$ core with hole filled with wax).

In processing the core images with wax in porous medium, data obtained during scanning of the solid wax piece were taken into account. After calibration of the tomograph, the main two stages of the experiment were started as follows:

1. scanning of the extracted and dried core sample to determine the initial pore volume and open porosity;

2. scanning the core after filtration of a wax-bearing solution with a decrease in temperature to determine the volume of pores clogged with wax. The Hounsfield unit for wax was taken into account during the reconstruction

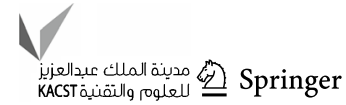




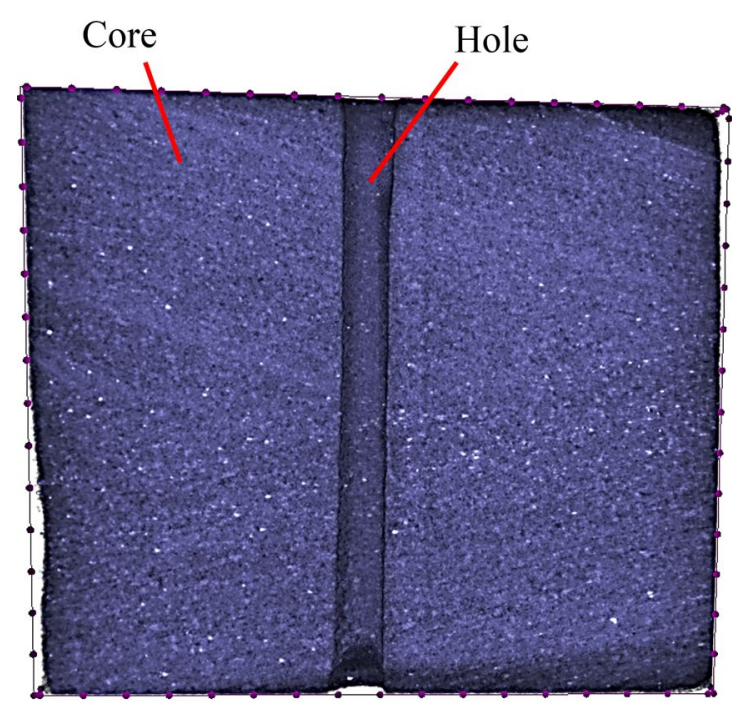

a

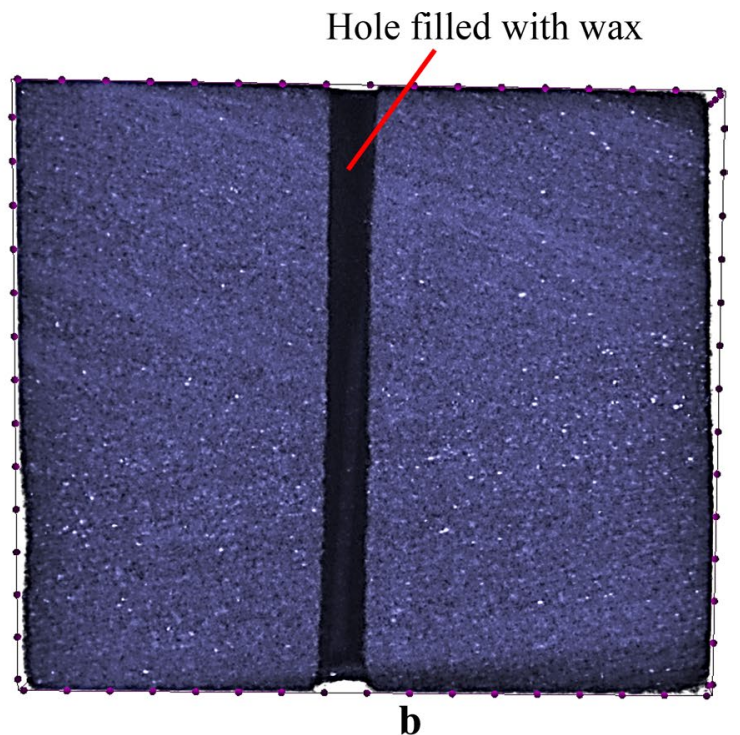

Fig. 1 Images of core with drilled hole (a empty hole, $\mathbf{b}$ hole filled with wax)

of images. The great difference between HU for wax $(-600 \mathrm{HU})$, water $(0 \mathrm{HU})$ and rock $(\approx 600 \mathrm{HU})$ allows to separate each phase in the images and determine the damage area.

The following flowchart was made to visualize the experimental procedure for measuring WAT (Fig. 2).

\section{Results and discussion}

The results of rheological studies are presented in Fig. 3. WAT, which was equal to $30^{\circ} \mathrm{C}$, was defined based on the dependence of the viscosity of a wax-bearing solution on temperature. It is worth noting that WAT in an open measuring system can differ from WAT of the same solution in the porous medium (Zlobin 2012; Zlobin and Yushkov 2013).

Figure 4 presents the results of the flooding experiment.

Figure 4 shows that the pressure gradient increases with a decrease in temperature. A slight increase in the pressure gradient, in the temperature range of $40-35^{\circ} \mathrm{C}$, is due to an increase in the solution viscosity. A sharp increase in the pressure gradient, in the temperature range of $35-33{ }^{\circ} \mathrm{C}$, indicates a decrease in core permeability, which can be caused by the formation of wax crystals in the pore volume.

The comparison of the above results of rheological and flooding experiments showed that for a wax-bearing solution, the formation of wax crystals in the pore volume of the rock is registered at a temperature of $3-4{ }^{\circ} \mathrm{C}$ higher than in an open measuring system. We suppose that this phenomenon is due to the fact that the filtration method has a significantly greater sensitivity compared to the viscometry.
The deflection point in the viscosity curve appears at a temperature, at which a lattice of wax begins to form in the studied solution. The wax crystallization since nucleation till the formation of a lattice can take a long time, depending on the shear stress and the temperature gradient. The pore volume of the core is represented by pore throats of various diameter, comparable to the size of wax nucleus. Therefore, stable wax crystal nuclei can already create blocks for fluid flow in the porous medium. Accordingly, wax crystallization by the filtration method is observed at a higher temperature.

Figure 5 shows two images (a-core segment with size of $0.6 \times 0.6 \times 1.2 \mathrm{~mm}$, light gray is rock, dark gray is pore space, b-light gray is rock, dark gray is pore space, red is wax).

To assess change in the pore volume of the core resulting from wax crystallization, a computed tomography was carried out. 3D models of the core were constructed before and after filtration of a wax-bearing solution, and open porosity of the sample was determined. The results of the study are presented in Fig. 5 and in Table 1.

From the data, presented in Table 1 and Fig. 5, it can be seen that after filtration a wax-bearing solution, with a decrease in temperature, open porosity of the core sample decreased from 9.0 to $2.1 \%$, that demonstrate a significant clogging the pore volume with wax.

For a more detailed analysis of the change in the pore volume of the core sample resulting from clogging with solids, distribution of pore throats by diameter before and after filtration was implemented. The results are presented in Fig. 6.

Figure 6 shows that $76.7 \%$ of the pore volume (in the range of pore diameters from 20 to $70 \mu \mathrm{m}$ ) is involved in the clogging with wax. Pores with a diameter comparable (or 

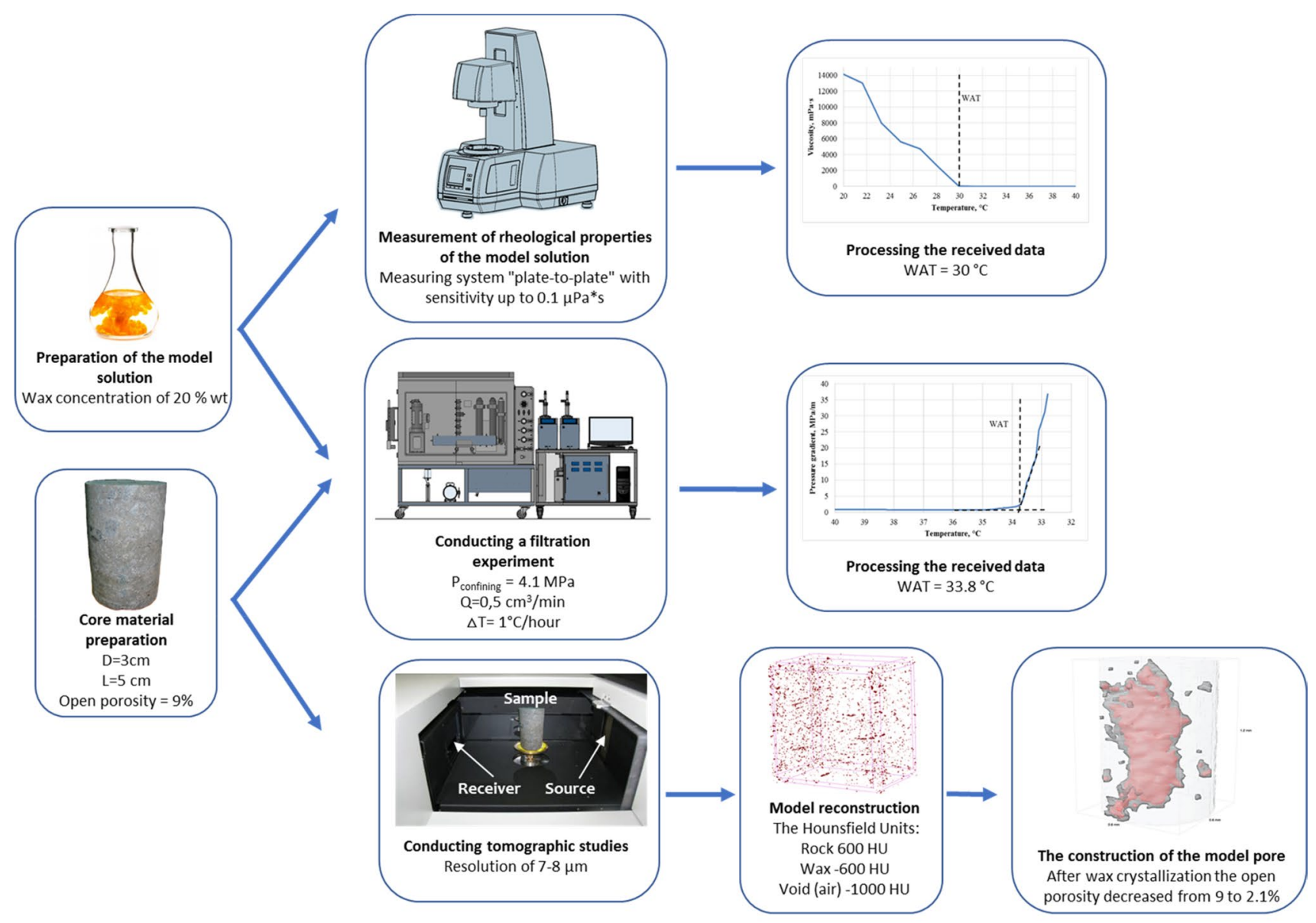

Fig. 2 The experimental procedure flowchart

Fig. 3 The dependence of the viscosity of a wax-bearing solution on temperature (viscosity curve)

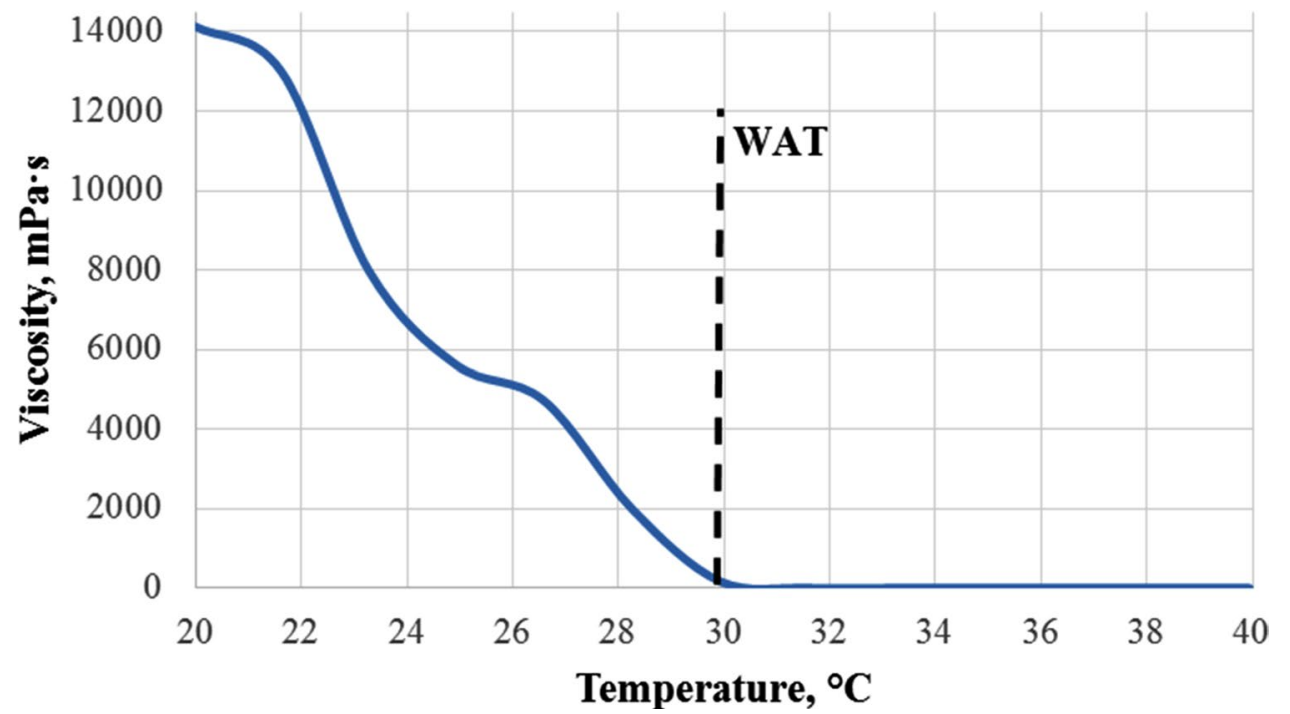

less) to the size of wax particles formed in kerosene are presumably clogged. In pores with a diameter slightly exceeding the size of wax particles, wax adsorption is observed.
The results of the study showed that large pores are less involved in the clogging, which is coherent with a number of studies (Shedid and Zekri 2006; Papadimitriou et al. 2007). 
Fig. 4 The dependence of the pressure gradient on temperature

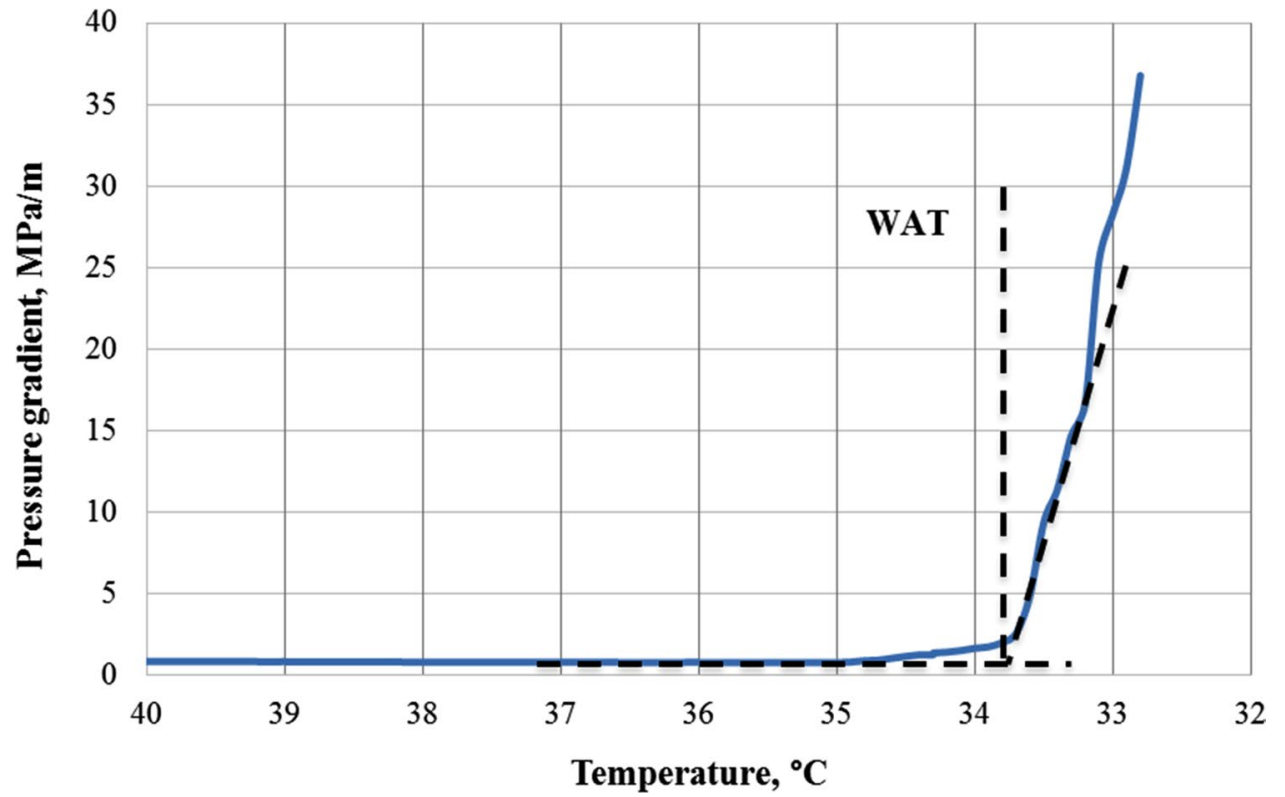

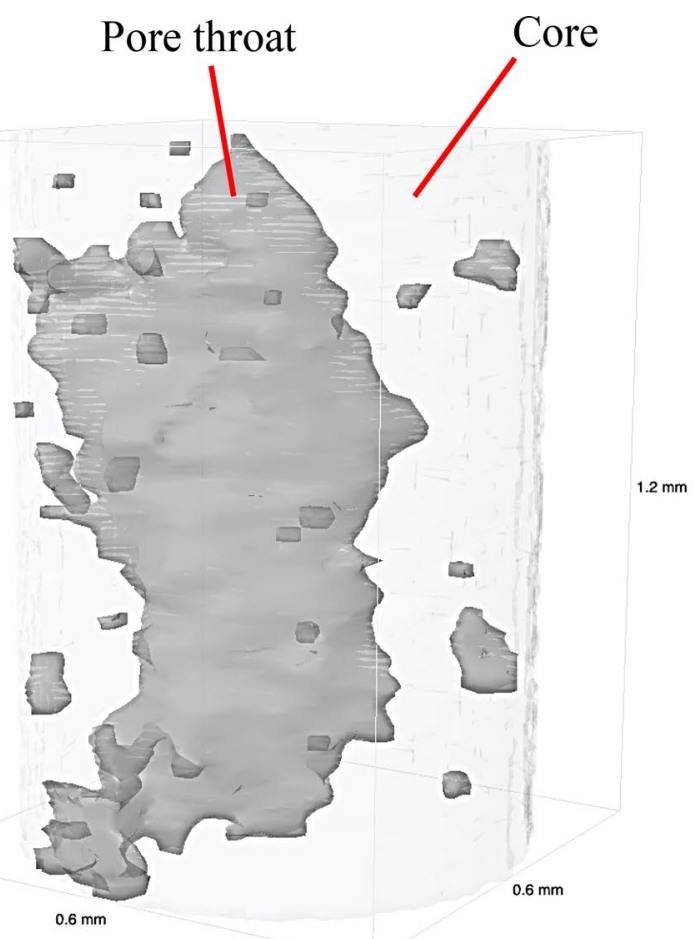

$\mathbf{a}$

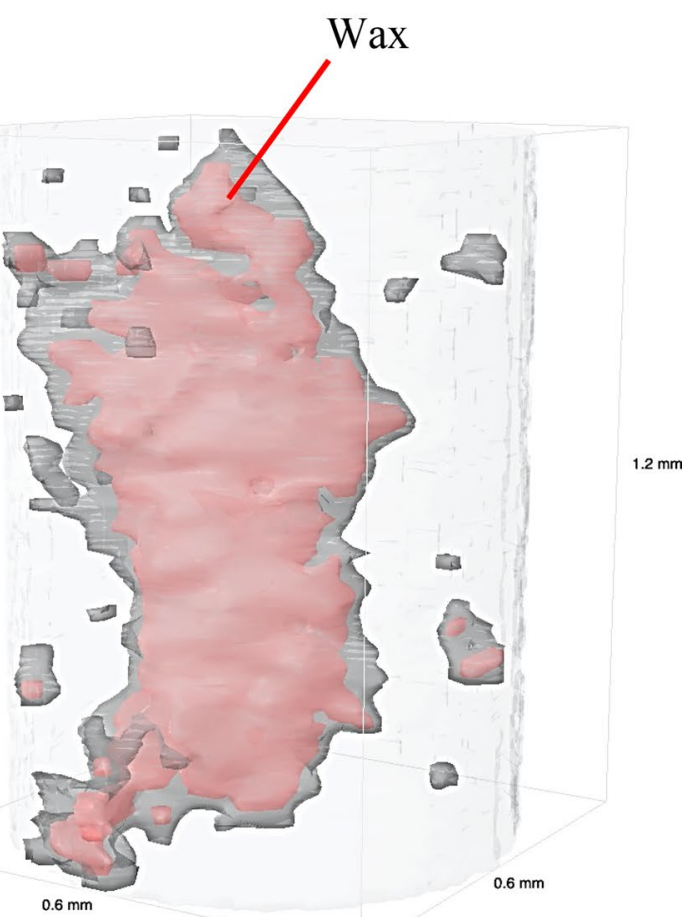

b

Fig. 5 3D models of the core segment (a the extracted and dried sample; $\mathbf{b}$ the sample after filtration of a wax-bearing solution with a decrease in temperature)

Table 1 The porosity of the core sample according to micro-computed tomography data

Parameter $\quad$ Extracted core sample Core sample after filtration with a decrease in temperature

Open porosity, \% 9.0

\section{Simulation of wax deposition in porous medium}

The generalized Navier-Stokes equations and Darcy's law were used to describe the flow of an incompressible fluid in the porous medium. At the external boundaries and rock surface, the normal and tangential velocity components were 


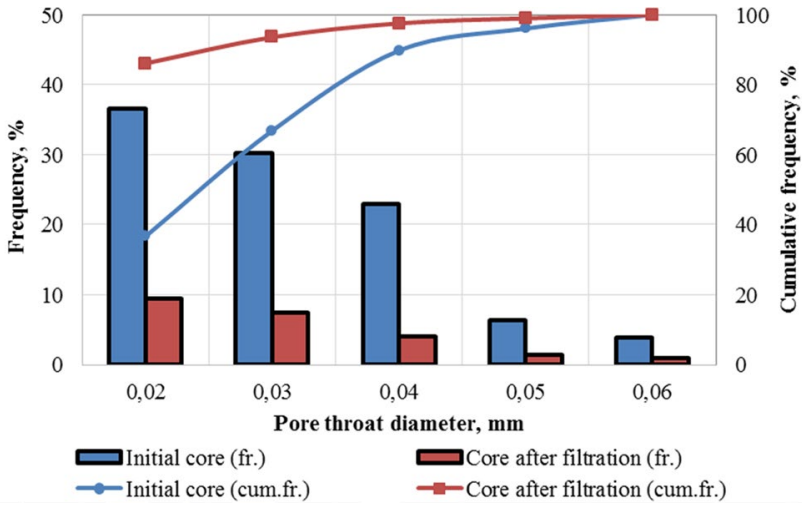

Fig. 6 Distribution of pore throats by diameter before and after filtration

taken equal to zero. In the dynamic model, a porous medium is represented by two continuously permeable and impermeable pathways. Fluid flow in a porous medium can be described by specifying a full porosity model. In those areas of the model where geometry of pore throats is too complicated, it is possible to set the resistance to flow coefficients. In this case, the model contains a diffusion term.

Generalized Darcy's law was used in the model:

$-\frac{\partial p}{\partial x_{i}}=\frac{\mu}{k} U_{i}+k_{\mathrm{IRF}} \frac{\rho}{2} U_{i}^{2}$,

where $\frac{\partial p}{\partial x_{i}}$ is the pressure gradient along the linear coordinate $x, \mathrm{~Pa} / \mathrm{m} ; \mu$ is fluid viscosity, $\mathrm{mPa} \mathrm{s} ; k$ is core permeability, $\mu \mathrm{m}^{2} ; U_{i}$ is fluid velocity components, $\mathrm{m} / \mathrm{s} ; \rho$ is fluid density, $\mathrm{kg} / \mathrm{m}^{3} ; k_{\mathrm{IRF}}$ is the inertial resistance factor.

The first term on the right-hand side of equation represents a viscous loss term and the second term on the righthand side of equation represents an inertial loss term. The inertial resistance factor can be represented by the following expression:
$k_{\mathrm{IRF}}=-\frac{2 \Delta P A^{2}}{\rho L Q^{2}}$

where $\Delta P$ is the pressure drop along the sample with a length of $L, \mathrm{~Pa} ; A$ is filtration area, $\mathrm{m}^{2} ; Q$ is the fluid volumetric flow rate, $\mathrm{m} / \mathrm{s}$.

In laminar flow through porous media, the pressure drop is typically proportional to velocity and the inertial resistance factor can be considered to be zero. So that, the porous media model then reduces to Darcy's law.

Two dynamic models (pore domains with reservoir properties) were built in a simulator using computed tomography data and tuned to the results of flooding experiments before and after wax deposition in the pore volume. Figures 7 and 8 show the flow velocity profiles in three sections of a core sample for two dynamic models, as well as streamlines. The direction of fluid flow coincides with the Y-axis.

Wax deposition causes reduction in hydraulic diameter of pores, correspondingly, porosity and permeability. Wax particles, adsorbed onto the surface of the pore throats, occupied part of the pore volume necessary for filtration, therefore, regions with high flow velocities decreased in size, and regions with minimum flow velocities increased compared to the initial sample (Fig. 8).

3D dynamic simulation using digital core makes it possible to quickly estimate the reservoir properties of the sample without conducting costly and time-consuming flooding experiments. Often there is not enough core material for proper justification of the well simulation technique, and standard laboratory techniques do not allow resimulation of filtration process after chemical or physical reactions in the core (for example, after acidizing), since the initial core structure is disturbed. The creation of a digital core allows reproducing the results of actual laboratory filtration experiments and generating new cases with new operating conditions, including conditions of irreversible change in the pore space of the core. In this case, a

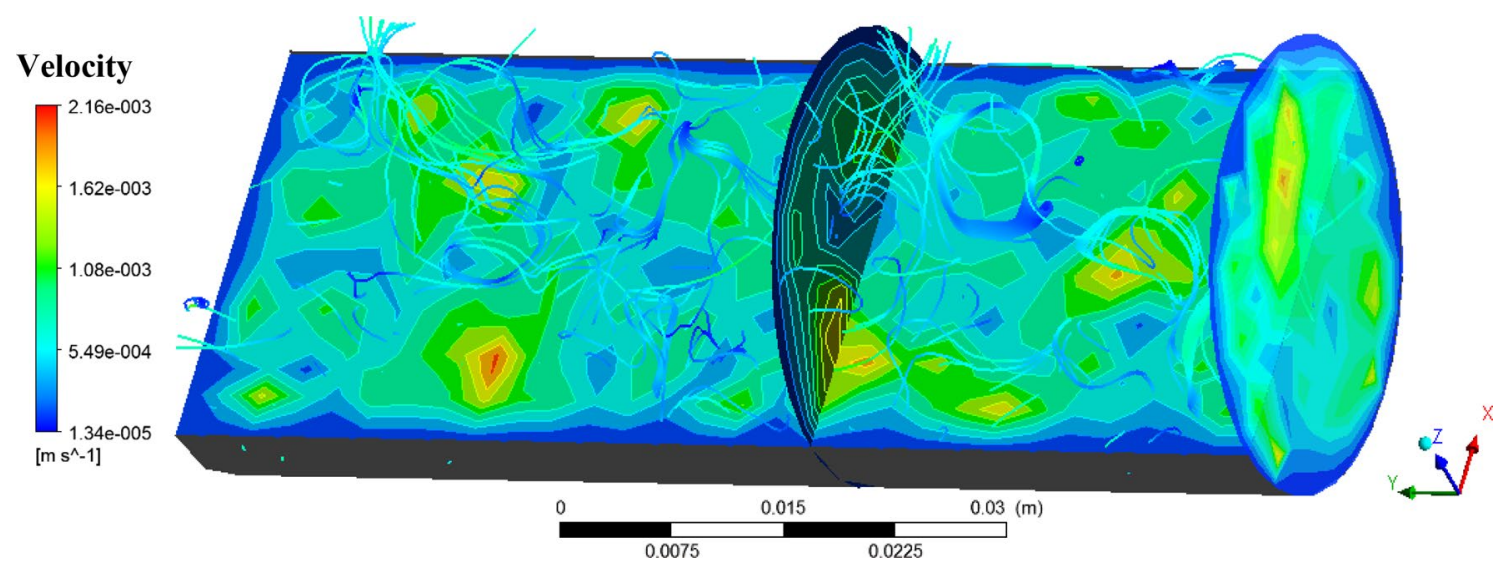

Fig. 7 Flow velocity profile across core sample before wax deposition 


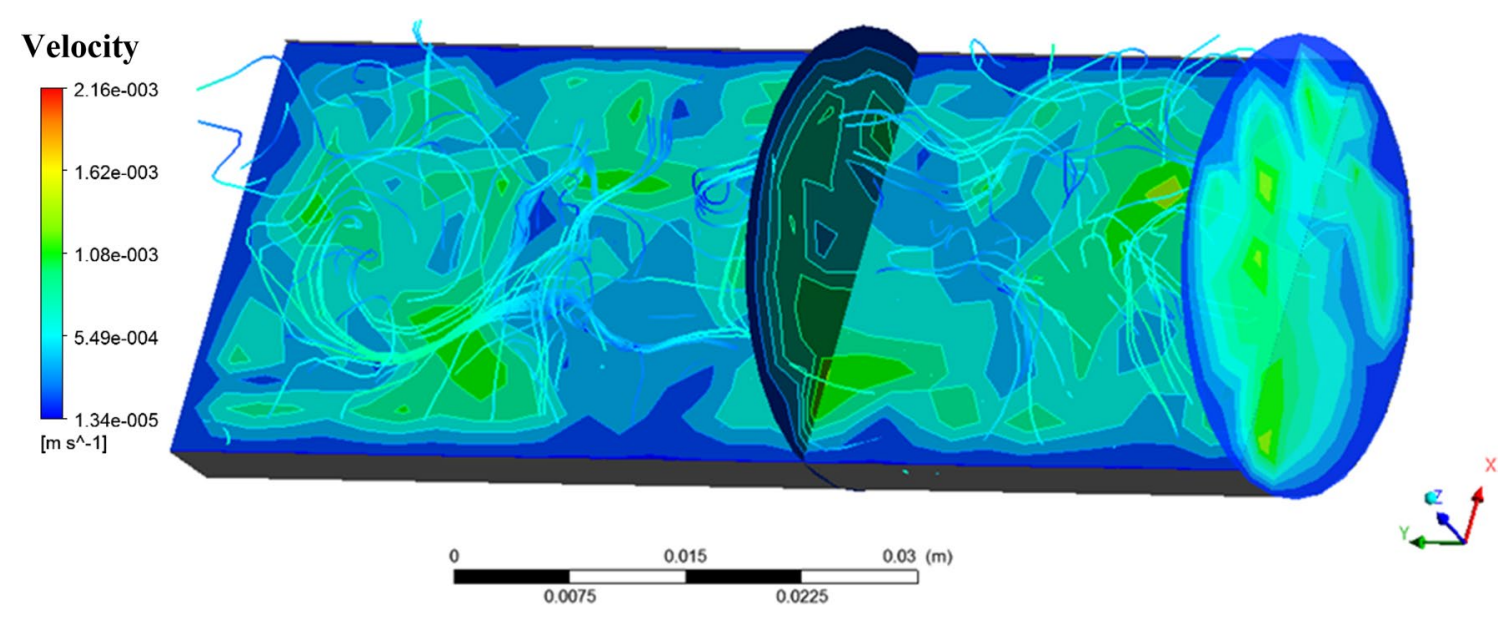

Fig. 8 Flow velocity profile across core sample after wax deposition

digital core, which can also be used when it is impossible to study the core by the filtration method (for example, unconsolidated rocks), is advantageously used.

\section{Calculation of temperature field during cold water injection}

The field operation with cold water injection can be accompanied by the change in the temperature field and cause phase transitions of wax in oil during production. To assess the possibility of wax crystallization in the pore volume of the reservoir, the authors calculated the change in the temperature field in the near-wellbore area of the injection well resulting from cold water injection by the example of the Romashkinskoye field. The calculations were based on the extended Lauwerier's concept (Lauwerier 1955, Saeid and Barends 2009). The concept resides in calculating heat transport in a reservoir with constant thickness, porosity and permeability. Lauwerier assumes that the vertical reservoir temperature gradient is neglected; the lower boundary of the reservoir is water and heat sealed, and the upper boundary is only water sealed. So, heat transfers in the reservoir just by convection and into the upper boundary by vertical conduction. The equations were proposed by Lauwerier for the calculation of the temperature distribution in the reservoir as a function of time and position during hot water injection into the injection well. Later, based on the Lauwerier's concept, Barends (2010) proposed to calculate the temperature field in hot reservoir during cold water injection into the injection well. The extended Lauwerier's concept for convective-conductive heat transfer with bleeding to adjacent layers was proposed by Saeid and Barends (2009). The extended Lauwerier solution is presented below (Saeid and Barends 2009):
$T=T_{1} \operatorname{erfc}\left[\frac{\xi}{2 \sqrt{\tau-\xi(1-\delta)}}\right] U[\tau-\xi(1-\delta)]$,

where $T$ is the reservoir temperature at distance $x(\mathrm{~m})$ from injection well, ${ }^{\circ} \mathrm{C} ; T_{1}$ is injected temperature, ${ }^{\circ} \mathrm{C} ; \operatorname{Erfc}(x)$ is complementary error function; $U(x)$ is the unit function.

$\xi=\frac{x h D^{\prime}}{v H^{2}}$,

$\tau=\frac{t h D^{\prime}}{H^{2}}$

$\delta=\frac{h D D^{\prime}}{v^{2} H^{2}}$

where $h$ is the heat capacity ratio; $D, D^{\prime}$ is the reservoir and the adjacent layer thermal diffusivity, respectively, $\mathrm{m}^{2} / \mathrm{s} ; v$ is heat convective velocity, $\mathrm{m} / \mathrm{s} ; t$ is time, $s ; H$ is reservoir height, $\mathrm{m}$.

The calculation results are presented in Fig. 9. The curves were plotted with reservoir properties of rocks, as well as field data accounted for.

The figure shows that in 1 year after the start of cold water injection, a decrease in reservoir temperature is observed at a distance of $280 \mathrm{~m}$ from the injection well. Upon 7 years of the injection well operation, the cold front will move to $600 \mathrm{~m}$. Calculation of the change in the thermal field in the nearwellbore area of the injection well over time has shown that it is possible to cool the reservoir with the considered reservoir properties to a temperature equal to WAT, and the cold front can reach, within 5-7 years, the producing wells, located at a distance of $600 \mathrm{~m}$ from the injection well and, accordingly, clogging of the porous medium with wax may take place. It is worth noting that the accuracy of the calculations is limited by the reservoir heterogeneity along the cross section, which 
Fig. 9 The calculated temperature field in the near-wellbore area of the injection well

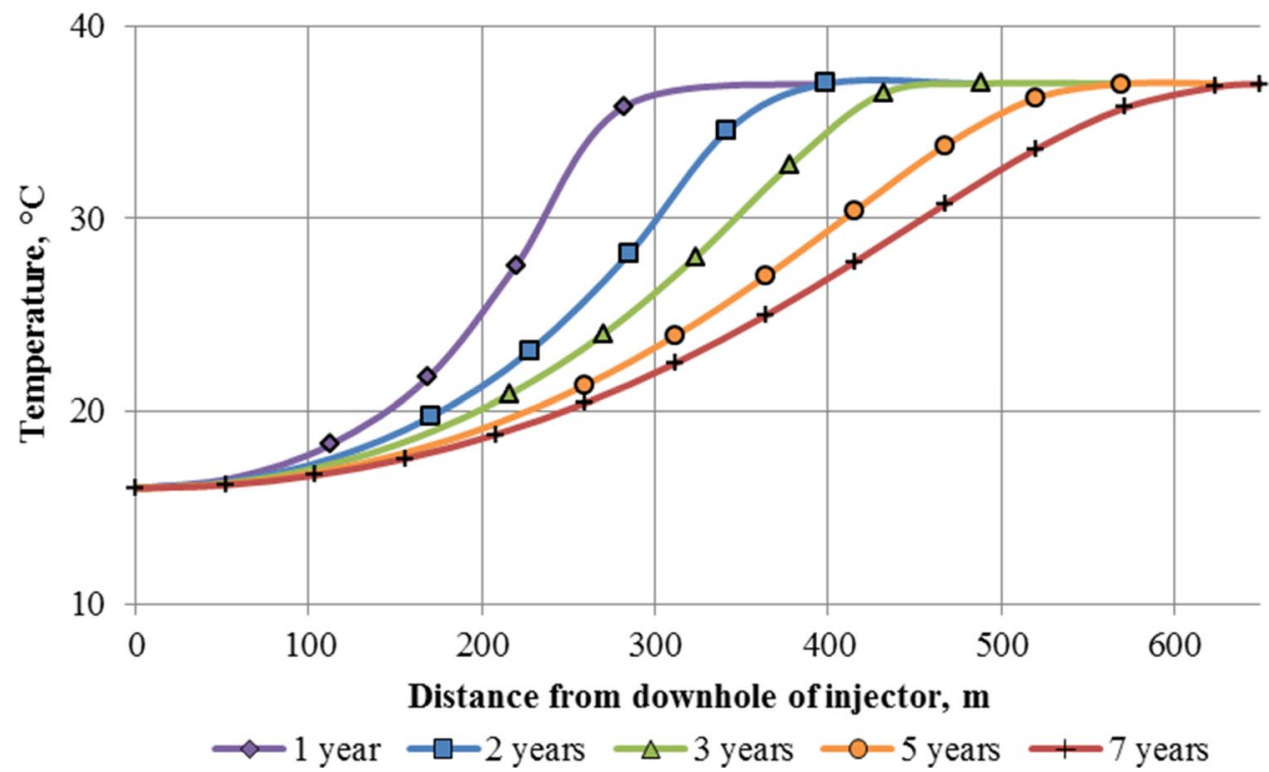

causes flushing the reservoir highly permeable areas with large volume of water and, accordingly, high cooling rates.

\section{Estimation of wax deposition in the reservoir}

At temperature below WAT, wax can be partially dissolved, suspended in oil, and partially adsorbed onto the surface of the pore throats. Wax solubility and precipitation can be described by the ideal-solution theory (Weingarten and Euchner 1988):

$x_{\mathrm{ws}}=x_{\mathrm{wl}} \exp \left[\frac{\Delta H}{R T}\left(1-\frac{T}{\mathrm{WAT}}\right)\right]$,

where $x_{\mathrm{ws}}$ is the molar fraction of wax precipitates in oil, unit fraction; $x_{\mathrm{wl}}$ is the molar fraction of wax dissolved in oil, unit fraction; $\Delta H$ is enthalpy of wax crystallization, $\mathrm{kJ} / \mathrm{mol} ; R$ is universal gas constant, $\mathrm{kJ} / \mathrm{mol} \mathrm{K} ; T$ is absolute temperature, $\mathrm{K}$; WAT is wax appearance temperature, $\mathrm{K}$.

During temperature decrease, the equilibrium in Eq. 7 moves toward wax crystallization. Equation 7 allows to assess the amount of wax that precipitates in oil, depending on temperature. To find the ratio of suspended wax to wax dissolved in oil at different temperatures, it is required to determine the enthalpy of wax crystallization. Struchkov and Rogachev (2017) obtained the dependence of WAT on the pressure and wax concentration in kerosene by a visual method. The results are presented in Fig. 10.

According to the authors, the dependence of WAT on pressure for wide range of wax concentrations in kerosene
Fig. 10 Pressure versus WAT for different wax concentration in kerosene (Struchkov and Rogachev 2017)

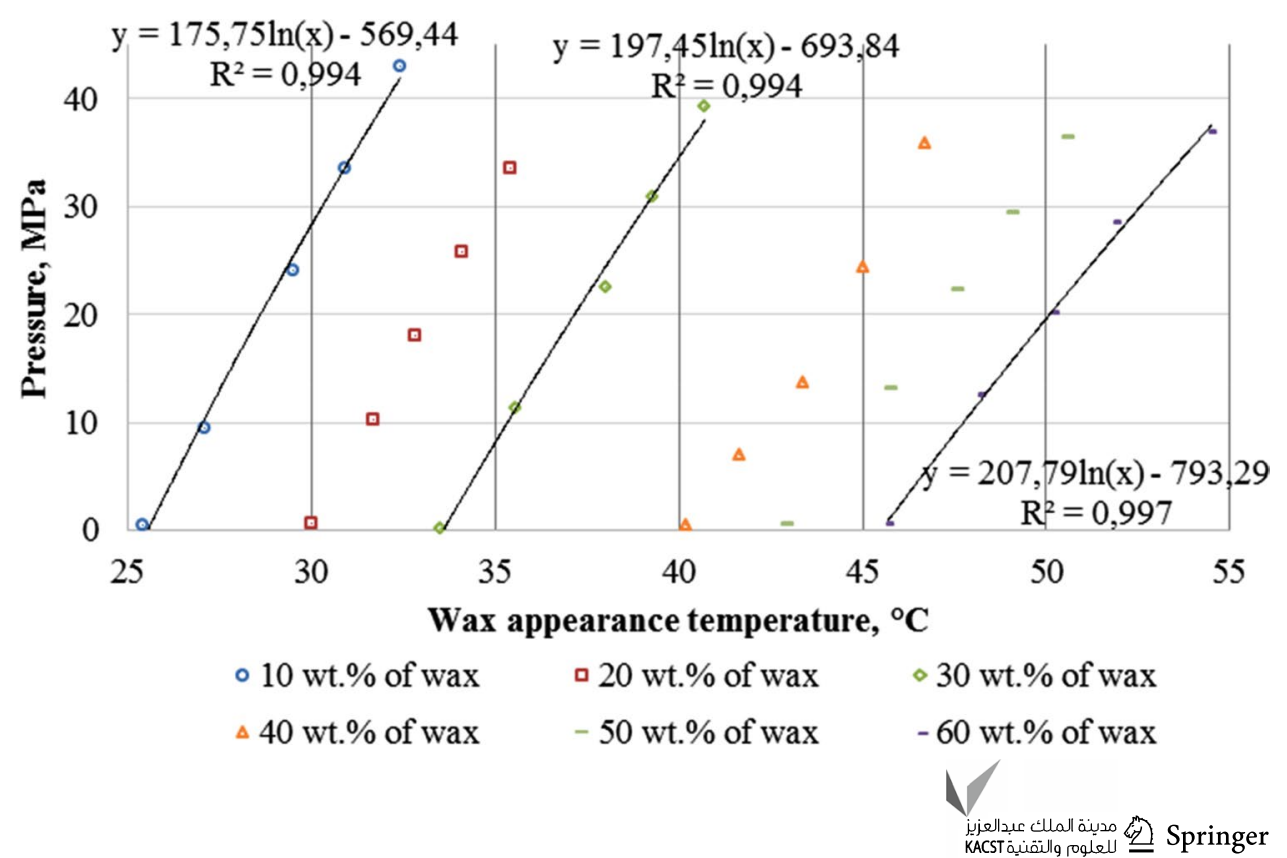


has a logarithmic trend and can be expressed by the following equation:

$P=P^{*}+k \ln \left(\frac{\mathrm{WAT}}{\mathrm{WAT}^{*}}\right)$,

where $P^{*}$ is ambient pressure, $\mathrm{MPa}$; $\mathrm{WAT}^{*}$ is the wax appearance temperature in the solution under ambient pressure, ${ }^{\circ} \mathrm{C}$; WAT is the wax appearance temperature in the solution under pressure $P,{ }^{\circ} \mathrm{C} ; k$ is a constant of the phase transition in the Clausius-Clapeyron equation.

Obtained data are completely confirmed by the thermodynamic Clausius-Clapeyron equation, describing the phase changes of matters during change in the pressure-temperature conditions (Sharma 2001). Such phase changes, named as first order transitions, are characterized by constant temperature and changes in entropy and volume. During wax crystallization, the heat is spent on creating a spatial lattice, formed by solid wax. As a result, the system acquires a more ordered state, which, according to the second law of thermodynamics, is accompanied by a decrease in the entropy. The packing density of wax molecules increases with increase pressure, distance of molecules in the solution decreases, free path of molecules reduces. The diffusion mechanism of wax crystallization comes into effect (more intense supply of wax molecules to the wax crystal nucleus takes place).

Based on the obtained results (Fig. 10), Struchkov and Rogachev (2018) constructed Van`t Hoff plots for pressure ranging from 0.1 to $35 \mathrm{MPa}$ and wax concentration in kerosene ranging from 10 to $60 \%$ (Fig. 11).

Transforming the Van`t Goff Eq. (1886) and Gibbs equation, the following equation was obtained (Ashbaugh et al. 2002):

$\ln \left(\frac{1}{x}\right)=\frac{\Delta H}{R T}-\frac{\Delta S}{R}$

where $x$ is molar fraction of wax in kerosene, unit fraction; $\Delta S$ is entropy of wax crystallization, $\mathrm{kJ} / \mathrm{mol} \mathrm{K}$.
The thermodynamic parameters of wax crystallization in kerosene can be obtained by processing the data of Fig. 11 using Eq. 9. Thus, the enthalpy of wax crystallization in kerosene at atmospheric pressure is $81.7 \mathrm{~kJ} / \mathrm{mol}$, which is consistent with the results obtained according to Paso et al. (2005). The deviation of calculated parameters may be due to inaccuracy when estimating the molecular weight of wax and kerosene, as well as to a high molar concentration of wax in kerosene, because Van`t Goff equation describes highly diluted solutions. In calculations, the molar weights of wax (with hydrocarbon number of $\mathrm{C}_{36}$ ) and kerosene equal to $506 \mathrm{~g} / \mathrm{mol}$ and $163 \mathrm{~g} / \mathrm{mol}$, accordingly, were used.

The wax mass balance can be described by the following equation (Wang et al. 1999):

$\frac{\partial}{\partial t}\left(\varphi S_{\mathrm{o}} C_{\mathrm{w}} \rho_{\mathrm{w}}+\varphi S_{\mathrm{o}} \rho_{\mathrm{o}} w_{\mathrm{wl}}\right)+\frac{\partial}{\partial x}\left(\rho_{\mathrm{o}} u_{\mathrm{o}} w_{\mathrm{ws}}+\rho_{\mathrm{o}} u_{\mathrm{o}} w_{\mathrm{wl}}\right)=-\rho_{\mathrm{w}} \frac{\partial E_{\mathrm{w}}}{\partial t}$,

where subscripts $w$ and $o$ represents wax and oil, respectively, $S_{\mathrm{o}}$ is the oil saturation, unit fraction, $C_{\mathrm{w}}$ is volume fraction of wax suspended in oil, unit fraction, $\rho_{\mathrm{o}}$ and $\rho_{\mathrm{w}}$ is density of oil and wax, respectively, $\mathrm{kg} / \mathrm{m}^{3}, w_{\mathrm{ws}}$ and $w_{\mathrm{wl}}$ are mass fraction of the suspended and dissolved wax in oil, respectively, unit fraction, $E_{\mathrm{w}}$ is volume fraction of wax adsorbed onto the surface of the pore volume, unit fraction, $u_{\mathrm{o}}$ is the superficial velocity of oil, $\mathrm{m} / \mathrm{s}$.

The superficial velocity can be derived from Darcy's law:

$u_{\mathrm{o}}=-\frac{k k_{\mathrm{ro}}}{\mu_{\mathrm{o}}} \frac{\partial P}{\partial x}$

where $k$ is the absolute permeability of the porous medium, $\mu \mathrm{m}^{2}, k_{\mathrm{ro}}$ is relative oil permeability, unit fraction, $\mu_{\mathrm{o}}$ is oil viscosity, $\mathrm{mPa} \mathrm{s}, P$ is pressure, $\mathrm{Pa}, x$ is linear coordinate, $\mathrm{m}$.

The solid wax deposition rate in the pore volume can be estimated using the model of wax deposition. In calculations, we used the deposition model developed by Gruesbeck and Collins (1982) for deposition of fine particles in porous medium:
Fig. 11 Van`t Hoff plots for different pressure (Struchkov and Rogachev 2018)

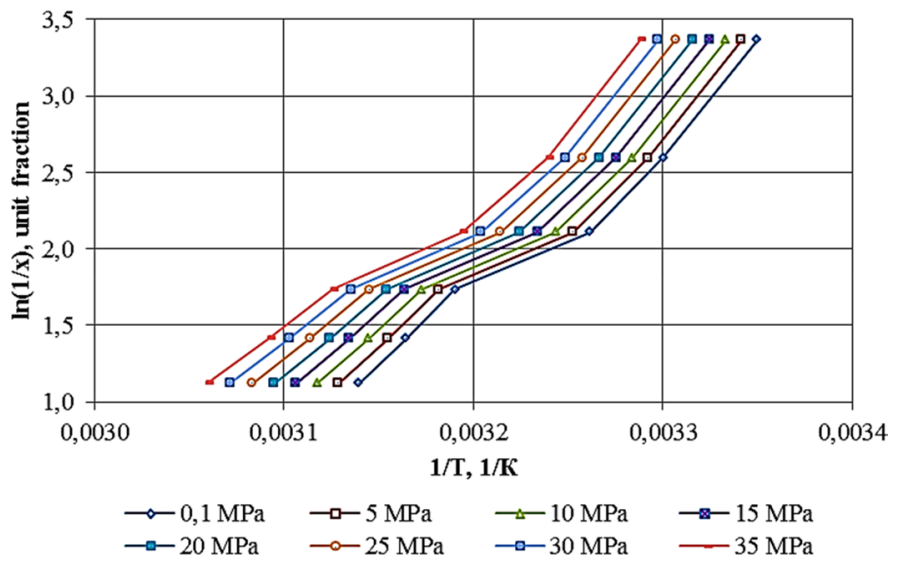


$\frac{\partial E_{\mathrm{w}}}{\partial t}=\overbrace{\alpha_{\mathrm{w}} S_{\mathrm{o}} C_{\mathrm{w}} \varphi}^{\text {adsorption }}-\overbrace{\beta_{\mathrm{w}} E_{\mathrm{w}}\left(v_{\mathrm{o}}-v_{\mathrm{cr}, \mathrm{o}}\right)}^{\text {desorption }}+\overbrace{\gamma_{\mathrm{w}} u_{\mathrm{o}} S_{\mathrm{o}} C_{\mathrm{w}}}^{\text {clogging }}$,

where $\frac{\partial E_{\mathrm{w}}}{\partial t}$ is the solid wax deposition rate, $\alpha_{\mathrm{w}}$ is the adsorption coefficient, $\beta_{\mathrm{w}}$ is the desorption coefficient, $v_{\mathrm{o}}$ is the interstitial velocity, $\mathrm{m} / \mathrm{s}, v_{\text {cro }}$ is the critical interstitial velocity, $\mathrm{m} / \mathrm{s}, \gamma_{\mathrm{w}}$ is the clogging rate coefficient.

$v_{o}=\frac{u_{o}}{\varphi}$.

The first term of expression 12 describes the rate of wax adsorption on the surface of pores. The second term describes the rate of wax desorption from the surface of pores, when the interstitial velocity is larger than the critical interstitial velocity. The third term describes pore throats clogging with wax.

The calculation procedure resides in assessing the number of pore volumes of oil, with suspended wax, pumped through the porous medium. The concentration of suspended wax in oil from the inlet to the outlet of the core sample is described by a power law. The amount of wax, deposited in the core, increases with growth in number of injected pore volumes. The calculation allows to assess the change in the reservoir properties during wax deposition in the pore volume, as well as to predict well operation conditions.

The adsorption and desorption coefficients can be estimated over the results of flooding experiments. At the first stage, the model solution is injected into the core at the interstitial velocity below the critical interstitial velocity determined empirically. Then concentration of suspended wax in solution outlet of the core is measured by available methods (nuclear magnetic resonance method Batsberg Pedersen et al. 1991, freezing wax from saturated hydrocarbons, extracted from solution via SARA fractionation Yang and Kilpatrick 2005), while the concentration inlet of the core remains constant. The concentration of wax in solution outlet of the core is lower than the inlet concentration as long as the wax is adsorbed onto the surface of the pore throats. As a result of the experiment, the following data are obtained: number of injected pore volumes of solution, concentration of suspended wax in solution outlet of the core over time, initial porosity of the core and duration of the experiment. To calculate the amount of wax deposited in the core sample, the following equations were used. Duration of the solution interaction with the core:

$t=\frac{\pi m D^{2} L}{4 Q} n$

where $m$ is porosity of the core, fraction; $D$ is diameter of the core, $\mathrm{cm}$; $L$ is length of the core, $\mathrm{cm} ; Q$ is flow rate, $\mathrm{cm}^{3} /$ $\min ; n$ is the number of injected pore volumes of solution.
The weight of wax deposited in the porous medium is a function of time and can be calculated in terms of wax mass balance by the following equation:

$m_{\mathrm{w}}(t)=\frac{1}{4} \pi m D^{2} L \rho\left(\omega_{i}-\int_{0}^{t_{\text {end }}} \omega(t) \mathrm{d} t\right)$

where $\rho$ is density of solution at the inlet of the core, $\mathrm{g} / \mathrm{cm}^{3}$.

At the next stage, the interstitial velocity is increased above the critical interstitial velocity and the experiment is carried out again. The concentration of suspended wax in solution outlet of the core becomes higher than the inlet concentration. The obtained data from the first and second stages are substituted into Eq. 12, making the clogging rate coefficient zero. Required adsorption and desorption coefficients are calculated by solving a set of equations. The more stages of the experiment, the more accurately the coefficients are estimated.

The instantaneous porosity is calculated as the difference between the initial porosity and the volume fraction of wax adsorbed onto the surface of the pore throats (Wang et al. 1999):

$\varphi=\varphi_{i}-E_{\mathrm{w}}$,

where $\varphi$ and $\varphi_{i}$ are the instantaneous and initial porosity, respectively, unit fraction.

Permeability takes the following form (Carman 1937; Kozeny 1927):

$k=k_{i}\left(\frac{\varphi}{\varphi_{i}}\right)^{3}\left(\frac{1-\varphi_{i}}{1-\varphi}\right)^{2}$,

where $k$ and $k_{i}$ are the instantaneous and initial permeability, respectively, $\mu \mathrm{m}^{2}$.

To assess the relative change in reservoir permeability, the permeability damage factor (Kdf) was introduced:

$\mathrm{Kdf}=1-\frac{k}{k_{i}}$

The calculating technique for wax deposition in the pore volume of rock is as follows:

According to the Lauwerier's concept, the thermal front distribution in the reservoir for the required forecast period is calculated (the calculation results are adjusted for the reservoir temperature monitoring data over time). Then, the thermal field around the producing well is compared with WAT. Thus, the area, wherein the pore volume is supposed to be clogged with wax crystals is estimated (the area around the well, where the temperature is below WAT, is estimated). Further calculations are made for this area. Based on Eq. 7, the amount of suspended wax crystals in oil, within the outlined area, is estimated depending on the reservoir temperature. The circular area around the well of radius $r$ is divided 
into radial sections of width dr (rings), wherein, based on the flow continuity rule, firstly the oil volume with suspended wax crystals is estimated depending on the initial production rate and time of the well operation. Time is also divided into dt intervals. Then, based on Eq. 12, taking into account the obtained adsorption and desorption coefficients (based on laboratory flooding experiments), the volume of wax crystals, deposited in the pore volume over time in each section of the reservoir, is calculated. Next, according to Eqs. 16 and 17 , the porosity and permeability changes, respectively, are estimated in each dr section around the producing well, depending on the operation time $(\mathrm{d} t)$. The calculations represent an iterative process. In the next time interval, the calculations are repeated until the required forecast time is reached. The shorter time intervals $(\mathrm{d} t)$ are associated with the higher forecast accuracy.

Taking into account the change in the temperature field resulting from cold water injection using wax deposition model, we obtained the dependence of the permeability damage factor in the near-wellbore of the producer Kdf on the well operation time, as well as oil production profile without and with wax deposition. The calculation results are given in Figs. 12 and 13.

The figure shows that after 1 year of production, an average permeability of the near-wellbore area will decrease by $28 \%$, due to the formation damage, caused by wax deposition.

The figure shows that a significant deviation of the production profile (with wax deposition) from oil production profile without wax deposition is observed after 1 year of the well operation.

The thermochemical method can be proposed as a remediation technique after wax deposition in the pore volume of the reservoir. The use of thermochemical reservoir stimulation methods is widespread in oil fields at a late stage of production, as well as in heavy oil fields. This technology is based on the use of binary solutions. As the active agent,

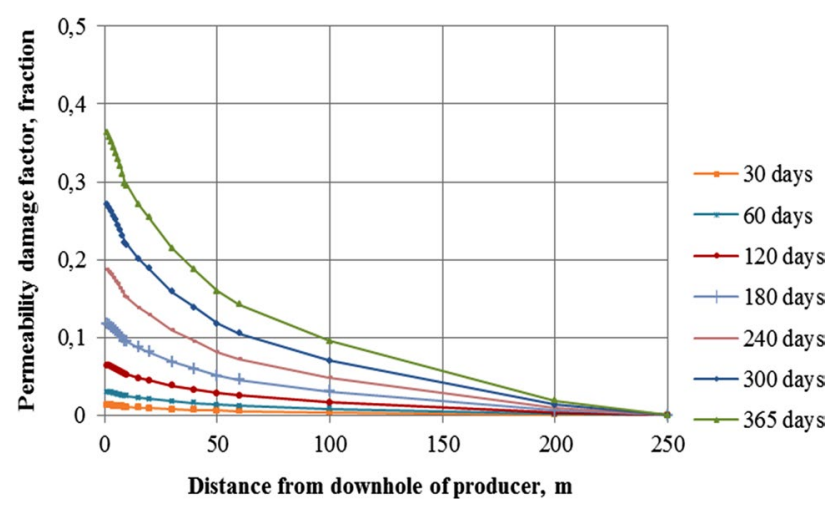

Fig. 12 The permeability damage factor along the radius (r) on the well operation time

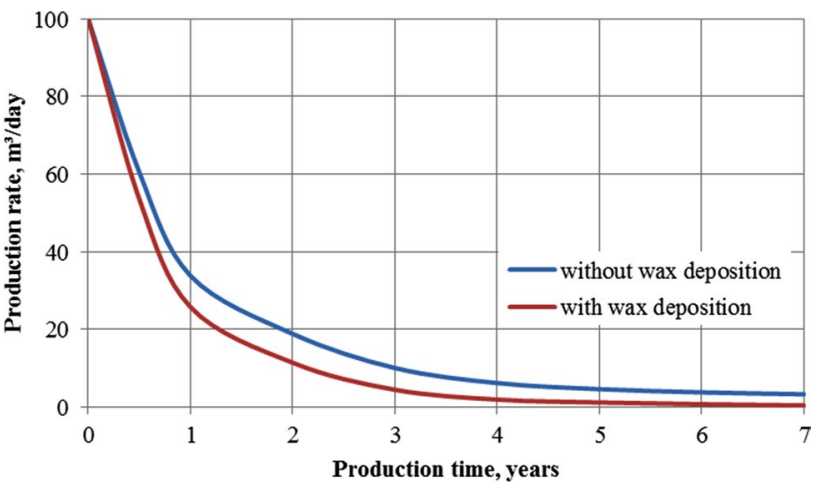

Fig. 13 Production rate without and with wax deposition

a combined mixture consisting of ammonium nitrate and sodium nitrite is often used. To treat the near-wellbore area, the reaction activator, flush fluid (water), active agent and displacement fluid (water) are injected step by step. As a result of interaction between components, a series of chemical reactions occurs accompanied by the release of large amounts of heat. The temperature created in the reservoir during the reaction will depend on the concentration of active agent, the injection rate, as well as on the residual oil saturation of rock (Kravchenko et al. 2018). The first two factors are controlled at the wellhead during treatment. Residual oil saturation can be controlled by well-logging operations in combination with a complex of laboratory studies. The availability of information on the reservoir saturation makes it possible to specify the time and place of the reaction, as well as to select more precisely the volume of the flush fluid separating the activator and the active agent. As a result, the rock in the treatment area is heated to a temperature, significantly exceeding the melting point of wax. Moreover, this technique is characterized by affordable cost compared to other thermal methods. The width of reservoir treatment depends on the volume of chemicals used and can reach first tens of meters, and the time of chemical reaction can be controlled by inhibitors (Zavolzhski et al. 2014; Khalil et al. 1997). When carrying out such treatment procedures, it is required to carefully control the transportation of chemicals and handling. They should not be allowed to penetrate into water reservoir or soil near oil fields. During heat-generating reaction, there is a sharp increase in both temperature and pressure, therefore, it is necessary to carefully control the wellbore and the cement sheath integrity, also to prevent the disaggregation of the producing formation top, since this can result to poisoning of groundwater.

In those cases when the radius of the clogged area exceeds the radius of the near-wellbore area treatment, hydraulic fracturing will contribute to remediate the connection between the well and the uninvaded formation area through a system of fractures. 
In the near-wellbore area of injection wells, wax deposition can be observed to a lowest extent. This is due to the fact that the cold front is far behind the flood front. As a result, only those areas that have already been flushed with several pore volumes of water will cool down to WAT. However, the pore volume in these areas will be saturated only with residual oil and water, therefore possible wax crystallization will not make blockage for water flow (Maloney and Osthus 2005).

Based on a post evaluation of the field production and calculations, it can be concluded that a decrease in well productivity could be associated with wax deposition in the pore volume resulting from injection of large volume of cold water and the reservoir cooling.

In oil fields with the use of the reservoir pressure maintenance systems, a decrease in reservoir temperature, due to the injection of cold water during the winter and the autumnspring period, is observed. The results of the paper showed that WAT in the pore volume of the reservoir is by $3-4{ }^{\circ} \mathrm{C}$ higher than in the open measuring system. Thus, during the field production, it is necessary to constantly control the thermal front in the reservoir to prevent the reservoir cooling below WAT (to prevent the clogging of pore volume of rock). Control the well operation conditions to minimize the risks associated with organic scales deposition in the field becomes substantiated if the field development monitoring is carried out, well survey and laboratory studies with calculations are provided simultaneously.

\section{Uncertainty analysis}

In the paper, an uncertainty analysis was carried out to estimate the relationship between the input parameters and the responses, and to determine heavy-hitters. For estimation, the one-level design, which allows one to study the sensitivity of the output variables based on the selected uncertainty parameter (the separated effect of each the input calculation parameters on the output parameters, which were the initial production rate and the cumulative oil production), was used. Therefore, a set of experiments was designed in accordance with the ranges of the input parameters. In the experiments, the minimum, maximum, and basic values of the input variables were used to generate response surfaces. Each of these cases was calculated using the technique presented in the paper. Other experimental design techniques have been discussed in greater detail by numerous scientists (Tartakovsky and Broyda 2011; Santoso et al. 2020; Li et al. 2011; Santoso et al. 2019). Based on the results of screening, tornado diagrams are constructed (Figs. 14 and 16).

Since the clogging rate coefficient is a combined parameter, the authors decided to use both the instantaneous clogging rate coefficient and the snowballing deposition constant in the uncertainty analysis. The clogging rate coefficient has the following expression:

$\gamma_{\mathrm{w}}=\gamma_{\mathrm{wi}}\left(1+\sigma E_{\mathrm{w}}\right)$,

where $\gamma_{\mathrm{wi}}$ is the instantaneous clogging rate coefficient, $\sigma$ is the snowballing deposition constant.

As is seen in the Fig. 14, the adsorption coefficient and the instantaneous clogging rate coefficient are the most influencing parameters on the initial production rate. The nomenclature of all variables is given in the paper.

Then, using Monte Carlo simulations, 10,000 cases were generated with different variations of the input parameters (the input parameters varied within the specified range). Based on the calculation results, a histogram, shown in Fig. 15, was plotted. In the figure it can be seen that the
Fig. 14 Tornado diagram showing the effect of the input parameters on the initial production rate

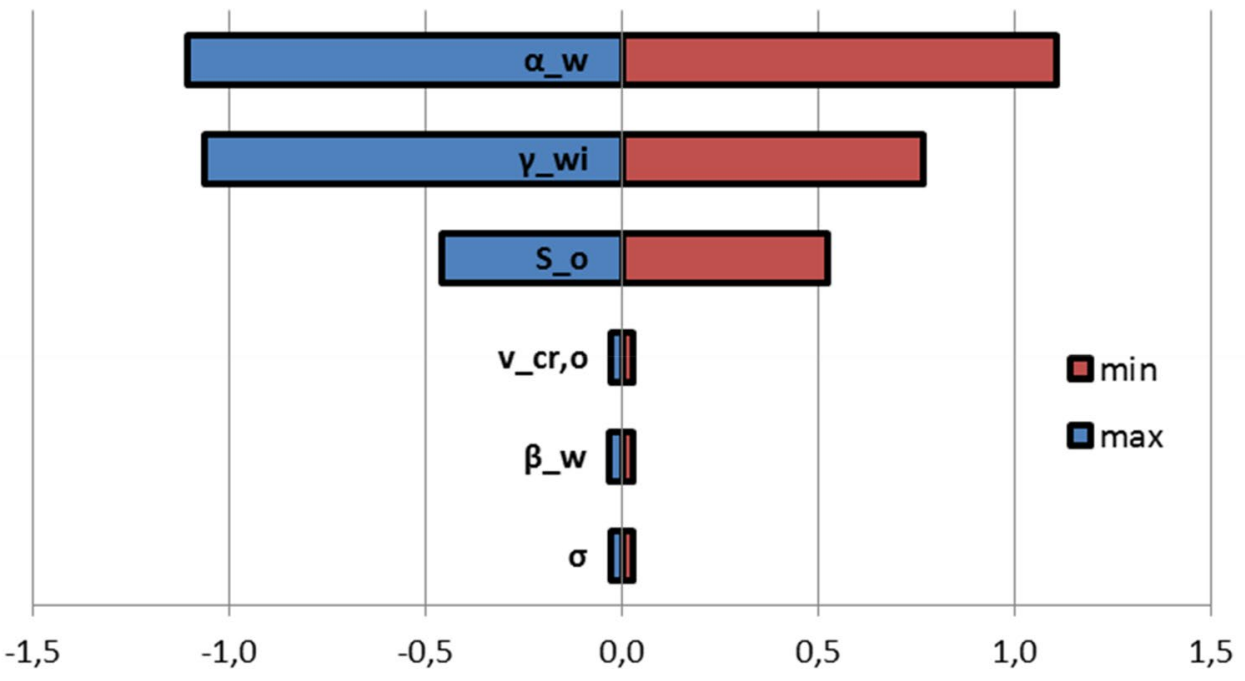

Effect on the initial production rate 
Fig. 15 Results of Monte Carlo simulations showing the initial production rate

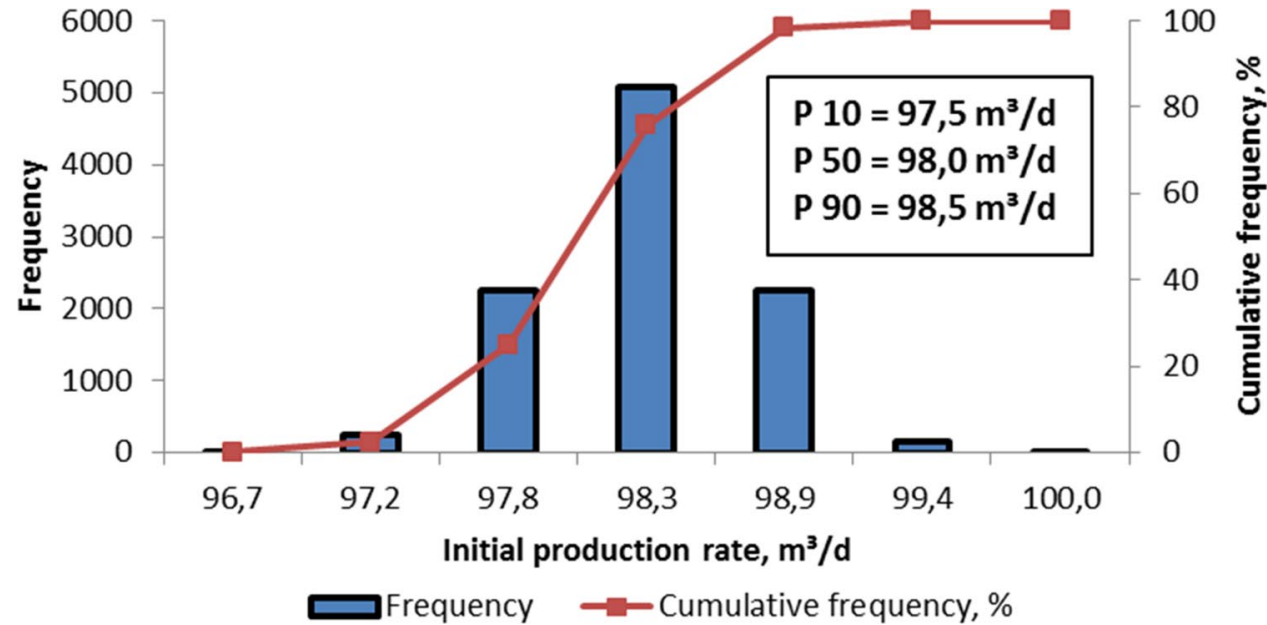

production rate range (from 97.5 to $98.5 \mathrm{~m}^{3} /$ day) falls within the confidence interval. The maximum production rate ( $100 \mathrm{~m}^{3} /$ day) corresponds to the case without wax deposition in the pore volume of rock.

The effect of the input variables on the cumulative oil production is presented in Fig. 16. In the figure it can be seen that the instantaneous clogging rate coefficient and the adsorption coefficient are also the most influencing parameters on the cumulative oil production.

As is seen in Fig. 17, the cumulative production falls within the confidence interval (from 29 to $39 \mathrm{Mm}^{3}$ ).

\section{Conclusions}

The results of rheology and flooding experiments showed that wax crystallization in the pore volume occurs at a temperature of $3-4{ }^{\circ} \mathrm{C}$ higher than in an open measuring system.
The results of computed tomography studies of a core sample, performed before and after filtration of a waxbearing solution with a decrease in temperature, showed that the open porosity decreased from 9.0 to $2.1 \%$, due to clogging the pore volume with wax. The pore throats with diameters from 20 to $70 \mu \mathrm{m}$ are involved in the clogging with wax.

The calculation results of production profiles using the porosity and permeability reduction models showed that in the field, a decrease in the productivity of producing wells could be caused by the formation damage, induced by wax deposition.

The obtained experimental data must be taken into account during the field production in case of the organic scales formation in the near-wellbore area of producing wells, to ensure more reliable prediction and efficient prevention of the formation damage.
Fig. 16 Tornado diagram showing the effect of the input parameters on the cumulative oil production

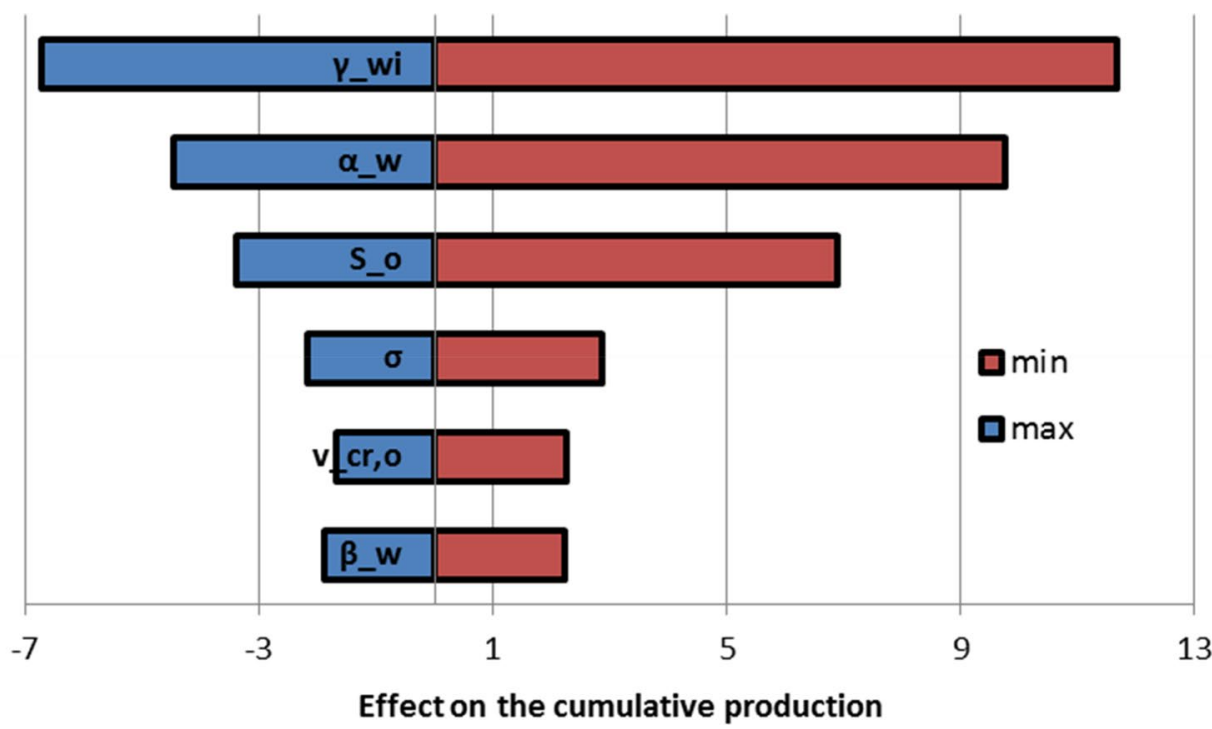


Fig. 17 Results of Monte Carlo simulations showing the cumulative oil production

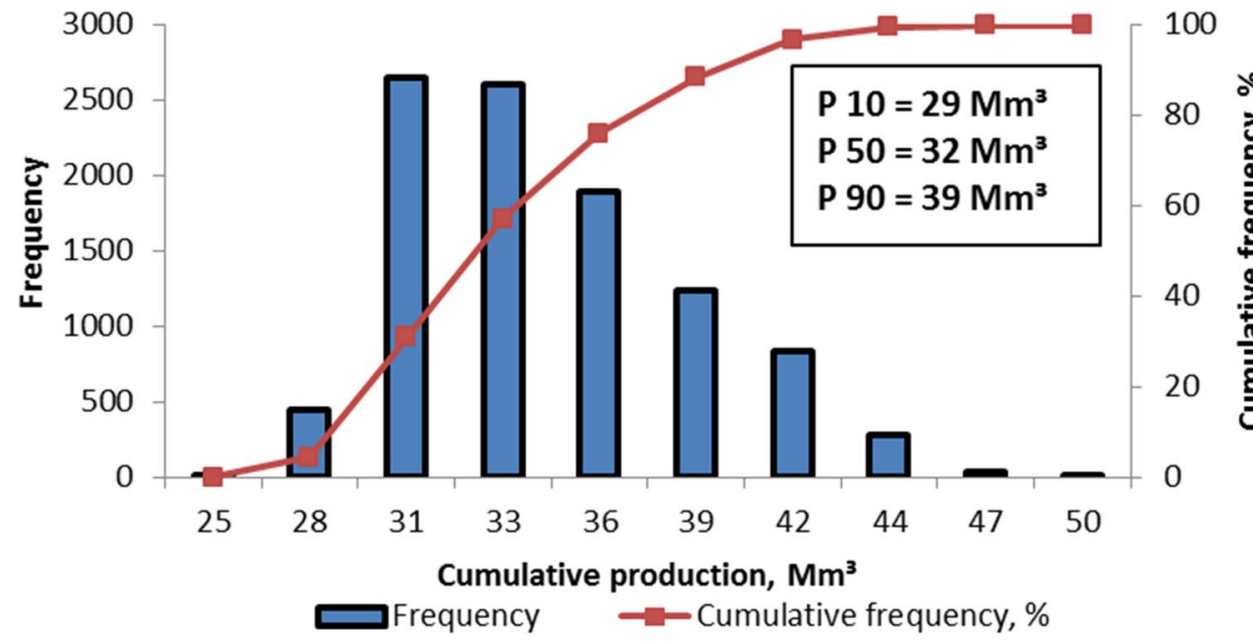

Acknowledgements We would like to thank Saint-Petersburg Mining University for providing laboratory equipment support and core samples for this research.

Open Access This article is licensed under a Creative Commons Attribution 4.0 International License, which permits use, sharing, adaptation, distribution and reproduction in any medium or format, as long as you give appropriate credit to the original author(s) and the source, provide a link to the Creative Commons licence, and indicate if changes were made. The images or other third party material in this article are included in the article's Creative Commons licence, unless indicated otherwise in a credit line to the material. If material is not included in the article's Creative Commons licence and your intended use is not permitted by statutory regulation or exceeds the permitted use, you will need to obtain permission directly from the copyright holder. To view a copy of this licence, visit http://creativecommons.org/licenses/by/4.0/.

\section{References}

Ashbaugh HS et al (2002) Interaction of paraffin wax gels with random crystalline/amorphous hydrocarbon copolymers. Macromolecules 35(18):7044-7053

Bacon MM, Romero-Zeron LB, Chong K (2009) Using cross-polarized microscopy to optimize wax-treatment methods. In: SPE annual technical conference and exhibition

Barends FBJ (2010) Complete solution for transient heat transport in porous media, following Lauwerier's concept. In: SPE annual technical conference and exhibition, Florence

Batsberg Pedersen W, Baltzer Hansen A, Larsen E, Nielsen AB, Roenningsen HP (1991) Wax precipitation from North Sea crude oils. 2. Solid-phase content as function of temperature determined by pulsed NMR. Energy Fuels 5(6):908-913

Borisov GK, Ishmiyarov ER, Politov ME, Barbaev IG, Nikiforon AA, Ivanov EN, Voloshin AI, Smolyanets EF (2018) Physical modeling of colmatation processes in the near-well bottom zone of Sredne-botuobinsky field. Part 2. Simulation of colmatation of a formation porous space by oil components. Oilfield Eng 12:64-67 (Russian)

Carman PC (1937) Fluid Flow through Granular Beds. Trans Inst Chem Eng 15:150-66
Chen H, Yang S, Nie X, Zhang X, Huang W, Wang Z, Hu W (2014) Ultrasonic Detection and analysis of wax appearance temperature of kingfisher live oil. Energy Fuels 28(4):2422-2428

Dos Santos JDS, Fernandes AC, Giulietti M (2004) Study of the paraffin deposit formation using the cold finger methodology for Brazilian crude oils. J Pet Sci Eng 45(1-2):47-60

Drzeżdżon J, Jacewicz D, Sielicka A, Chmurzyński L (2018) Characterization of polymers based on differential scanning calorimetry based techniques. TrAC Trends Anal Chem 110:51-56

Elsharkawy AM, Al-Sahhaf TA, Fahim MA (2000) Wax deposition from Middle East crudes. Fuel 79(9):1047-1055

Ferris SW, Cowles HC Jr, Henderson LM (1931) Composition and crystal form of the petroleum waxes. Ind Eng Chem 23(6):681-688

Gruesbeck C, Collins RE (1982) Entrainment and deposition of fine particles in porous media. Soc Pet Eng J 22(06):847-856

Huang Z, Zheng S, Fogler HS (2016) Wax deposition: experimental characterizations, theoretical modeling, and field practices. CRC Press, Boca Raton

Ijeomah CE, Dandekar AY, Chukwu GA, Khataniar S, Patil SL, Baldwin AL (2008) Measurement of wax appearance temperature under simulated pipeline (dynamic) conditions. Energy Fuels 22(4):2437-2442

Jiang Z, Hutchinson JM, Imrie CT (2001) Measurement of the wax appearance temperatures of crude oils by temperature modulated differential scanning calorimetry. Fuel 80(3):367-371

Jiang B, Ling QIU, Xue LI, Shenglai YANG, Ke LI, Han CHEN (2014) Measurement of the wax appearance temperature of waxy oil under the reservoir condition with ultrasonic method. Pet Explor Dev 41(4):509-512

Karan K, Ratulowski J, German P (2000) Measurement of waxy crude properties using novel laboratory techniques. In: SPE annual technical conference and exhibition. Society of Petroleum Engineers

Kozeny J (1927) Uberkapillare Leitung Des Wassersim Boden. Ber Wien Akd, p 271

Khalil CN, Rocha NO, Silva EB (1997) Detection of formation damage associated to paraffin in reservoirs of the Reconcavo Baiano, Brazil. In: International symposium on oilfield chemistry. Society of Petroleum Engineers

Kok MV, Létoffé J-M, Claudy P, Martin D, Garcin M, Volle J-L (1996) Comparison of wax appearance temperatures of crude oils by differential scanning calorimetry, thermomicroscopy and viscometry. Fuel 75:787-790

Kravchenko MN, Dieva NN, Lishchuk AN, Muradov AV, Vershinin VE (2018) Dynamic modeling of thermochemical treatment of

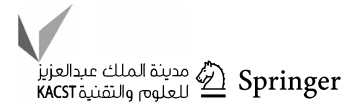


low permeable kerogen-containing reservoirs. Georesursy $=$ Georesources 20(3):178-185

Lauwerier HA (1955) The transport of heat into an oil layer caused by the injection of hot fluid. J Appl Sci Res A5(2-3):145-150

Li H, Sarma P, Zhang D (2011) A comparative study of the probabilistic-collocation and experimental-design methods for petroleumreservoir uncertainty quantification. SPE J 16(2):429-439

Maloney D, Osthus A (2005) Effects of paraffin wax precipitation during cold water injection in a fractured carbonate reservoir. Petrophysics 46(5):354-360

Mezzomo RF, Rabinovitz A (2001) Reservoir paraffin precipitation: the oil recovery challenge in Dom João field? J Can Pet Technol 40(6):46-53

Mingareev RSh, Vahitov GG, Sultanov SA (1968) Influence of cold water injection on the field development and oil recovery of Romashkin reservoir. Oil Ind J 11:26-30 (Russian)

Muslimov RH, Grajfer VI, Baziv VF (1968) The temperature field of Romashkin reservoir and the influence of cold water injection on the field development and oil recovery. Oil Ind J 11:31-35 (Russian)

Newberry ME, Barker KM (1985) Formation damage prevention through the control of paraffin and asphaltene deposition. In: SPE annual technical conference and exhibition. Society of Petroleum Engineers

Orodu OD, Tang Z (2014) The performance of a high paraffin reservoir under non-isothermal waterflooding. Pet Sci Technol 32(3):324-334

Papadimitriou NI, Romanos GE, Charalambopoulou GC, Kainourgiakis ME, Katsaros FK, Stubos AK (2007) Experimental investigation of asphaltene deposition mechanism during oil flow in core samples. J Petrol Sci Eng 57(3):281-293

Paso K et al (2005) Paraffin polydispersity facilitates mechanical gelation. Ind Eng Chem Res 44(18):7242-7254

Paso K, Kallevik H, Sjoblom J (2009) Measurement of wax appearance temperature using near-infrared (NIR) scattering. Energy Fuels 23(10):4988-4994

Rocha NO, Khalil CN, Leite LCF, Bastos RM (2003) A thermochemical process for wax damage removal. In: SPE annual technical conference and exhibition. Society of Petroleum Engineers

Saeid S, Barends FBJ (2009) An extension of Lauwerier's solution for heat flow in saturated porous media. In: Proceedings of the COMSOL conference, Milan, COMSOL

Santoso R, Hoteit H, Vahrenkamp V (2019) Optimization of energy recovery from geothermal reservoirs undergoing re-injection: conceptual application in Saudi Arabia. SPE-195155-MS

Santoso R, Torrealba V, Hoteit H (2020) Investigation of an improved polymer flooding scheme by compositionally-tuned slugs. Processes 8(2):1-20

Sharma BK (2001) Engineering chemistry, vol 5. Krishna Prakasan Media (P) Ltd, Meerut

Shedid SA, Zekri AY (2006) Formation damage caused by simultaneous sulfur and asphaltene deposition. SPE Prod Oper 21(01):58-64

Shepherd JE, Nuyt CD, Lee JJ, Woodrow JE (2000) Flash point and chemical composition of aviation kerosene (Jet A). Graduate
Aeronautical Laboratories California Institute of Technology Pasadena. Explosion Dynamics Laboratory Report FM99-4

Sherman AM, Geiger AC, Smith C, Taylor LS, Hinds J, Stroud P, Simpson GJ (2019) Stochastic differential scanning calorimetry by nonlinear optical microscopy. Anal Chem 92:1171-1178

Struchkov IA, Rogachev MK (2017) Wax precipitation in multicomponent hydrocarbon system. J Pet Explor Prod Technol 7(2):543-553

Struchkov IA, Rogachev MK (2018) The challenges of waxy oil production in a Russian oil field and laboratory investigations. J Pet Sci Eng 163:91-99

Tartakovsky DM, Broyda S (2011) PDF equations for advectivereactive transport in heterogeneous porous media with uncertain properties. J Contam Hydrol 120-121:129-140

Tronov VP, Guskova IA (1999) Mechanism of the organic scales formation at the last stage of the field development. Oil Ind J 4(1999):24-25 (Russian)

Uba E, Ikeji K, Onyekonwu M (2004) Measurement of wax appearance temperature of an offshore live crude oil using laboratory light transmission method. In: Nigeria annual international conference and exhibition

Van't Hoff JH (1886) Louis de l'équilibre chimique dans l'état dilué, gazeux ou dissous. PA Norstedt et Söner. Arch Neerl Sci Exact Nat 20:239-302

Vicky Kett, Simon Gaisford, Peter Haines (2016). Principles of thermal analysis and calorimetry. Royal Society of Chemistry, p. 280

Wang Y, Civan F, Strycker AR (1999) Simulation of Paraffin and Asphaltene Deposition In Porous Media. SPE International Symposium on Oilfield Chemistry. Society of Petroleum Engineers, Houston, Texas, pp 57-66

Weingarten JS, Euchner JA (1988) Methods for predicting wax precipitation and deposition. In: SPERE, pp 121-132

Yang X, Kilpatrick P (2005) Asphaltenes and waxes do not interact synergistically and coprecipitate in solid organic deposits. Energy Fuels 19(4):1360-1375

Yusupova TN, Barskaya EE, Ganeeva YuM, Romanov GV, Amirkhanov II, Hisamov RS (2016) Identification of wax deposits in the bottom-hole formation zone and wellbore in reducing of the pressure. Oil Ind J 1:39-41 (Russian)

Zavolzhski V, Burko V, Idiatulin A, Basyuk B, Sosnin V, Demina T, Ilyun V, Kashaev V, Sadriev F (2014) Thermo-gas-generating systems and methods for oil and gas well stimulation. U.S. Patent Application No. 14/090,928

Zlobin AA (2012) Analysis of phase transitions in the pore space paraffin reservoir rocks. Bull Perm Natl Res Polytech Univ Geol Pet Min Eng 5:47-56 (Russian)

Zlobin AA, Yushkov IR (2013) NMR study of petroleum paraffins in the porous medium of reservoir rocks. Bull Perm Natl Res Polytech Univ Geol 1(18):81-90 (Russian)

Publisher's Note Springer Nature remains neutral with regard to jurisdictional claims in published maps and institutional affiliations. 